\title{
Lorentz-Abraham-Dirac and Landau-Lifshitz equations of motion and the solution to a relativistic electron in a counterpropagating laser beam
}

\author{
Arthur D. Yaghjian๑* \\ Electromagnetics Research, 115 Wright Road, Concord, Massachusetts 01742, USA
}

(Received 18 August 2021; accepted 1 November 2021; published 29 November 2021)

\begin{abstract}
Beginning with a critical examination of the Lorentz-Abraham (LA) classical equation of motion for an extended charge and the closely related Lorentz-Abraham-Dirac (LAD) equation of motion for a massrenormalized point-charge, the Landau-Lifshitz (LL) approximate solution to the LAD equation of motion is determined for an electron subject to a counterpropagating linearly or circularly polarized plane-wave pulse with an arbitrarily shaped envelope. A convenient three-vector formulation of the LL equation is used to derive closed-form expressions for the velocities and associated powers of the electron directly in terms of the time in the laboratory frame. The three-vector formulation also reveals definitive criteria for the LL solution to be an accurate approximation to the LAD equation of motion and for the LL solution to reduce to the solution of the Lorentz force equation of motion that ignores radiation reaction. Semiclassical analyses are used to obtain simple conditions for determining the regimes where the quantum effects of either Compton electron scattering by the incident photons or electron recoil produced by the emitted photons is significant. It is proven that the LL approximation becomes an inaccurate solution to the LAD equation of motion only for large enough electron velocities and plane-wave intensities that quantum recoil effects on the electron can greatly alter the classical solution. Comparisons are made with previously published analytical and numerical solutions to the LL equation of motion for the velocity of an electron in a counterpropagating plane wave.
\end{abstract}

DOI: 10.1103/PhysRevAccelBeams.24.114002

\section{INTRODUCTION}

The deceleration of relativistic electrons by intense counterpropagating optical laser beams has produced $\mathrm{X}$-rays and, more recently, $\gamma$-rays in the laboratory [1-9]. Although the detailed theoretical determination of $\mathrm{X}$-ray and $\gamma$-ray production by the interaction of highenergy electrons with intense optical lasers may require the inclusion of quantum effects, a hybrid approach that incorporates quantum corrections into a classical solution can predict reasonable results $[10,11]$. Because the classical Lorentz-Abraham-Dirac (LAD) equation of motion does not have a closed-form solution to the problem of an electron in a plane wave, nor is it amenable to a numerical solution, especially when many charges are involved [12], the more readily solvable Landau-Lifshitz (LL) approximation to the LAD equation of motion has become the classical equation of choice for this problem within much of the physics community [11-14].

\footnotetext{
a.yaghjian@comcast.net
}

Published by the American Physical Society under the terms of the Creative Commons Attribution 4.0 International license. Further distribution of this work must maintain attribution to the author(s) and the published article's title, journal citation, and DOI.
Remarkably, Di Piazza [15,16] and later Hadad et al. [17] have derived a closed-form solution to the LL approximate equation of motion for the problem of a plane-wave pulse scattered by a moving electron. These authors use a four-vector formulation of the LL equation of motion to determine the solution for the velocity components of the electron in terms of the retarded time parameter $\xi=\omega(t+z / c)$, where $\omega$ is the angular frequency of the plane wave, $t$ is the time, $c$ is the speed of light, and $z$ is the time-dependent longitudinal coordinate of the electron (opposite the direction of propagation of a counterpropagating plane wave). The four-vector solution has also been rederived by Ruijter et al. [18] for an electron that is initially at rest. To obtain numerical results for the velocity components as functions of time $t$, expressions for the proper time $\tau$ in terms of $\xi$ are found in Ref. [17] and then the time $t$ is found in terms of $\tau(\xi)$ by numerically integrating the relativistic factor $\gamma(\tau)$ with respect to the proper time $\tau$.

In the present paper, the problem of the moving electron illuminated by linearly and circularly polarized counterpropagating plane-wave laser-beam pulses is revisited and solved directly with the three-vector electromagnetic field formulation of the LL approximate equation of motion. This approach has the advantage of maintaining transparency of the three-vector electromagnetic fields and velocities as well as the envelope function of the plane-wave pulse throughout 
the solution. In addition, the three-vector formulation reveals an explicit closed-form expression for the time $t$ in terms of the retarded time parameter $\xi$ without having to deal with the proper time $\tau$. It also facilitates the derivation of a simple useful formula for the error in the radiated power introduced by the LL approximation.

The explicit closed-form expressions for the velocity components and relativistic factor as well as the radiated, kinetic, Schott, and total-supplied power are evaluated numerically for linearly and circularly polarized, uniform and sinusoidal-envelope plane-wave pulses with a laserstrength parameter $a_{0}=100$ and an initial electron relativistic factor $\gamma_{0}=1000$ in order to compare with the numerical results given in Ref. [17] for the velocity components and relativistic factor. These large laser intensities and electron energies can produce measurable $\gamma$-ray radiation $[7,8]$. For this high-speed, high-intensity problem, it is found that the LL approximate solution predicts a radiated power that agrees very closely with the exact LAD expression for the radiated power evaluated with the approximate LL velocity and acceleration componentsa result also predicted by the formula derived for the error in the LL radiated power. Despite the erroneous irreversibility of the Schott power introduced by the LL approximate solution, this irreversible Schott power is found nonetheless to be nearly equal to the reversible Schott power in the LAD equation of motion. These close agreements in radiated and Schott powers in the LL and LAD solutions strongly confirm the high accuracy of the $\mathrm{LL}$ approximation to the LAD solution for these particular high-speed-electron and high-intensity-laser results.

Interestingly, the radius of the computed scattering cross section of the high-speed electron in the counterpropagating high-intensity laser is much closer to the Compton wavelength than the classical electron radius, which is on the order of the radius of the Klein-Nishina scattering cross section for Compton scattering of photons from the electron. Also, as one might expect, the observed rapid changes in the power radiated and relativistic factor near the beginning of the uniform (rectangular-envelope) laserbeam pulse are greatly reduced by the sinusoidal-envelope pulse that begins continuously from a value of zero fields.

For relativistic electrons in high-intensity optical laser fields, quantum effects may appreciably alter the classical results [11,19-24]. Therefore, a concise semiclassical determination is provided for the conditions on laser intensity and electron energies for deciding the importance of the three quantum effects that can significantly change the motion and radiation of the electron, namely quantumvacuum electron-positron pair production, Compton scattering of the incident photons, and electron quantum recoil from photon emission ("inverse Compton scattering") [1]. In addition, the $a_{0}-\gamma-\omega$ region of validity for the LL solution to be an accurate approximation to the LAD solution is determined by substituting the LL approximate solution into the exact expressions for the radiation power and momentum in the LAD equation of motion. Taken together, these conditions conclusively show that the LL approximation is an accurate solution to the LAD equation of motion except in the region of high enough values of the product $a_{0} \gamma$ that quantum recoil effects of the electron can dominate the solution. Nevertheless, in the region where the LAD equation is accurate but the LL equation is not, the LAD equation, if solvable, could prove useful as an initial classical solution for incorporating quantum effects. Two conditions are also found for this LL solution to be approximately equal to the Lorentz force (LF) solution (no radiation reaction). One of these two conditions reveals that the LF solution is never adequate and radiation reaction is always required if the electron has been subject to the plane-wave LF for a long enough time.

Though it has been more than a century since Lorentz and Abraham derived the classical equation of motion for an extended charged sphere, there continues to be considerable discussion and uncertainty in the literature concerning some of the more subtle aspects of the equation of motion and its derivation, specifically, the $4 / 3$ factor in the inertial mass term of the self force, the discrepancy between the kinetic power obtained from the self-force integral and from the self-power integral (as well as the relationship of this discrepancy to Poincaré stresses), the renormalization of the infinite mass of the classical extended charged sphere to a finite value as its radius is allowed to approach zero (to obtain the LAD equation of motion), and the noncausality (preacceleration and predeceleration) that arises in an otherwise wellbehaved solution to the LAD equation of motion.

Consequently, the paper begins with a critical review of the derivation of the LA equation of motion, explaining the root cause and remedy for the noncausality, the $4 / 3$ factor and Poincaré stresses, and the one remaining inconsistency introduced by renormalizing the mass of the charge as its radius approaches zero. The LL approximate solution is then derived simply and straightforwardly from a convenient three-vector form of the LAD equation of motion. The derivation manifestly separates the LL approximate radiation momentum-energy from the LL approximate Schott acceleration momentum-energy, the latter of which is no longer perfectly reversible in the LL approximate solution.

\section{THE LA AND LAD EQUATIONS OF MOTION}

An updated version of the LA equation of motion for an extended moving charge [[25-26] app. 7], [27] can be derived by evaluating the electromagnetic self-force on a relativistically contracting, nonrotating spherical insulator with a uniform surface charge (the Lorentz model), separating the radiation reaction force from the force needed to change the electromagnetic momentum of the accelerating charged sphere, then adding this radiation reaction force to the externally applied force in the relativistic version of Newton's second law of motion $[28,29]$. The resulting equation of motion in rationalized 
mksA (SI) units can be written in three-vector form as [[28] Eq. 7.12(a)]

$$
\begin{aligned}
\mathbf{F}_{\mathrm{ext}}(t)= & \left(m_{\mathrm{es}}+m_{\mathrm{ins}}\right) \frac{d}{d t}(\gamma \mathbf{u}) \\
& -\frac{e^{2}}{6 \pi \epsilon_{0} c^{3}}\left\{\frac{d}{d t}\left[\gamma^{2} \dot{\mathbf{u}}+\frac{\gamma^{4}}{c^{2}}(\mathbf{u} \cdot \dot{\mathbf{u}}) \mathbf{u}\right]\right. \\
& \left.-\frac{\gamma^{4}}{c^{2}}\left[|\dot{\mathbf{u}}|^{2}+\frac{\gamma^{2}}{c^{2}}(\mathbf{u} \cdot \dot{\mathbf{u}})^{2}\right] \mathbf{u}\right\}+\mathbf{O}(a)
\end{aligned}
$$

where $\mathbf{F}_{\text {ext }}(t)$ is the externally applied force (usually the LF), $\mathbf{u}(t)$ is the velocity of the center of the sphere, and the overdots indicate time derivatives of the velocity $(\dot{\mathbf{u}}=d \mathbf{u} / d t)$. The Lorentz relativistic factor is given by $\gamma=\left(1-u^{2} / c^{2}\right)^{-\frac{1}{2}}$, where $u=|\mathbf{u}|$. The electrostatic mass of the spherical shell with total charge $e$ and radius $a$ is

$$
m_{\mathrm{es}}=\frac{e^{2}}{8 \pi \epsilon_{0} a c^{2}},
$$

with $c$ the free-space speed of light, $\epsilon_{0}$ the permittivity of free space, and $m_{\mathrm{ins}}$ is the mass of the uncharged insulator. The electrostatic mass is obtained by combining the electrostatic energy of formation, $\mathcal{W}_{\mathrm{es}}=e^{2} /\left(8 \pi \epsilon_{0} a\right)$, of the spherical shell of charge with the Einstein mass-energy relation, $\mathcal{W}_{\mathrm{es}}=m_{\mathrm{es}} c^{2}$. Lorentz first derived the rest-frame equation of motion, $\mathbf{F}_{\mathrm{ext}}=m_{\mathrm{em}} \dot{\mathbf{u}}-e^{2} /\left(6 \pi \epsilon_{0} c^{3}\right) \ddot{\mathbf{u}}+\mathbf{O}(a)$, with $m_{\mathrm{em}}=4 m_{\mathrm{es}} / 3$ in his 1892 paper [25]. ${ }^{1}$ For a charged sphere moving with arbitrary velocity, Abraham was the first to derive the radiation-reaction parts of the force and power equations of motion in Eqs. (1) and (10b) below, respectively, as seen in his 1905 book [[27] Sec. 15]. The $\mathbf{O}(a)$ terms in the LA equation of motion in Eq. (1) are negligible under the rest-frame conditions $[28,29]$

$$
\frac{2 c}{3 a}\left|\sum_{n=3}^{\infty}\left(\frac{-2 a}{c}\right)^{n} \frac{1}{n !} \frac{d^{n} u^{\beta}(t)}{d t^{n}}\right| \ll\left|\frac{d u^{\beta}}{d t}\right| \ll \frac{c^{2}}{a}
$$

\footnotetext{
${ }^{1}$ The electromagnetic mass found from the electromagnetic momentum of the spherical shell of charge is $4 / 3$ times the electrostatic mass. This $4 / 3$ factor has been the subject of much discussion since the time of Lorentz and Abraham that continues to the present day [30]. Contrary to what is often stated in the literature, not only were Lorentz and Abraham unconcerned with this $4 / 3$ factor in their original work because it was done before Einstein's 1905 papers on special relativity and the mass-energy relation, but also this factor was not removed by Poincare in his analysis that determined there must be an extra term in the power equation of motion of the Lorentz contracting model of the charged sphere to account for the work done during contraction by the internal stresses that hold the charge onto the sphere [28]. Schwinger, in a paper devoted to the 80th birthday of Dirac, showed that there was no need for the electromagnetic and electrostatic masses to be equal because in the presence of charge-current the electromagnetic stress-momentum-energy tensor is not divergenceless [31].
}

$$
\frac{a}{c}\left|\frac{d^{2} u^{\beta}}{d t^{2}}\right| \ll\left|\frac{d u^{\beta}}{d t}\right|,
$$

where $u^{\beta}$ is a rectangular component of the velocity vector $\mathbf{u}$.

The first term on the right-hand side of Eq. (1) is the reversible kinetic acceleration (time rate of change of the kinetic momentum). The second $d / d t$ term in Eq. (1) is the reversible radiation-reaction acceleration often referred to as the Schott acceleration [32]. The remaining radiationreaction term on the right-hand side of Eq. (1) is the irreversible momentum radiated by the charge per unit time.

Two problems impede the solution to the LA equation of motion in Eq. (1). The first problem is the unknown contribution from the $\mathbf{O}(a)$ terms in Eq. (1) and the second problem is that the solutions to Eq. (1) that have zero velocities in the remote past and accelerations going to zero after the external force is turned off exhibit a noncausality near points in time where the external force is not an analytic function of time, such as when the external force is first applied (preacceleration) and when the external force is turned off (predeceleration). It can be shown that this noncausality occurs even if the $\mathbf{O}(a)$ terms are taken into account [[28] Chap. 8].

The root cause of the noncausality in the LA equation of motion is traced in Refs. [28] and [29] to the assumption in the derivation of Eq. (1) that the velocities at the retarded times $t^{\prime}=t-\Delta t_{a}$, where $\Delta t_{a} \approx 2 a / c$, can be expanded in a Taylor series about the present time $t$. This assumption requires that the externally applied force $\mathbf{F}_{\text {ext }}(t)$ be an analytic function of time in a complex $\Delta t_{a}$-radius neighborhood about each time $t$ on the real $t$ axis, an assumption that cannot be satisfied for a time $\Delta t_{a}$ following the point in time that the externally applied force is first applied or turned off. More generally, for externally applied forces that are complex functions of time about the real $t$ axis except for a finite number $N$ of nonanalytic points of time, the LA equation of motion in (1) is valid for all time except during the transition time intervals of duration $\Delta t_{a}$ following the nonanalytic points in time. ${ }^{2}$

\footnotetext{
${ }^{2}$ Abraham noted that "Because the internal force is determined by the velocity and acceleration existing in a finite interval preceding the affected point in time, such a progression [power series] is always possible when the movement is continuous and its velocity is less than the speed of light. ... [The series in the equation of motion] will converge more poorly the closer the movement approaches a discontinuous movement and the velocity approaches the speed of light. ... It fails completely for discontinuous movements" [[27] Sec. 23]. Schott also concludes that "the approximation [used to obtain the LA equation of motion] fails during an interval of time, which is comparable with the time required by an electromagnetic wave to pass across the electron and includes the instant at which the discontinuity occurs" [[33,34] p. 283]. More recently, Valentini [35] noted this problem with the derivation of the LA equation of motion near nonanalytic points of time of the applied force, but his proposed solution to the problem did not allow changes in velocity across the transition intervals and thus violated conservation of energy [36].
} 


\section{A. LA equation of motion modified with transition forces}

During (and only during) these small $\Delta t_{a}$ transition time intervals following the nonanalytic points in time of the external force, there have to be effective "transition forces", $\mathbf{f}_{a}(t)=\sum_{n=1}^{N} \mathbf{f}_{a n}(t)$, that remove the preacceleration (or predeceleration) from the original equation of motion and that modify the LA equation of motion in Eq. (1) to give

$$
\begin{aligned}
\mathbf{F}_{\mathrm{ext}}(t)+\mathbf{f}_{a}(t)= & \left(m_{\mathrm{es}}+m_{\mathrm{ins}}\right) \frac{d}{d t}(\gamma \mathbf{u}) \\
& -\frac{e^{2}}{6 \pi \epsilon_{0} c^{3}}\left\{\frac{d}{d t}\left[\gamma^{2} \dot{\mathbf{u}}+\frac{\gamma^{4}}{c^{2}}(\mathbf{u} \cdot \dot{\mathbf{u}}) \mathbf{u}\right]\right. \\
& \left.-\frac{\gamma^{4}}{c^{2}}\left[|\dot{\mathbf{u}}|^{2}+\frac{\gamma^{2}}{c^{2}}(\mathbf{u} \cdot \dot{\mathbf{u}})^{2}\right] \mathbf{u}\right\}+\mathbf{O}(a) .
\end{aligned}
$$

It was shown in Refs. [28] and [29] that, remarkably, the transition forces in this relativistically covariant modified LA equation of motion in Eq. (4) can be chosen to eliminate the preacceleration/deceleration while maintaining momentum-energy conservation, provided the following restframe inequality is satisfied for the radius $a$ small enough that $\left|m_{\text {ins }}\right| / m_{\text {es }} \ll 1$

$$
\frac{a\left|\Delta \mathbf{F}_{\mathrm{ext}}^{n}\right|}{m_{\mathrm{es}} c^{2}} \ll 1
$$

where, for an externally applied force that changes slowly on either side of the transition interval in a time duration on the order of $a / c$, the value of $\Delta \mathbf{F}_{\mathrm{ext}}^{n}$ is approximately equal to the difference in the rest frame of the externally applied force across the $n$th transition interval. ${ }^{3}$ The modification by these uncoupled transition forces also requires that the transition intervals be separated in time by an amount on the order of $a / c$ or greater.

In addition to the inequality in Eq. (5), it is shown in Refs. [28,29] that relativistic (Born) rigidity of the sphere requires a condition on the rest-frame jumps in velocity across the transition intervals, namely

$$
\frac{\left|\Delta \mathbf{u}_{n}^{0}\right|}{c} \ll 1,
$$

which remains a required condition across the transition intervals even as $a \rightarrow 0$ and the mass is renormalized to a finite value $m$.

For the unrenormalized electrostatic mass $m_{\mathrm{es}}$, the inequality in Eq. (5) is implied by the rest-frame equation of motion and the second inequality in Eq. (3a) required for

\footnotetext{
${ }^{3}$ More generally, $\Delta \mathbf{F}_{\text {ext }}^{n}$ is approximately equal to the rest-frame difference (of the externally applied force across the nonanalytic transition point in time $\tau_{n}$ ) averaged over the proper time from $\tau_{n}$ to $\tau_{n}+\tau_{e}$ [[28] Eq. 8.73(a)]. $\tau_{e}$ is defined in Eq. (8).
}

the $\mathbf{O}(a)$ terms to be negligible. Thus, the modified LA equation of motion in Eq. (4) is a causal equation of motion that satisfies momentum-energy conservation with the $\mathbf{O}(a)$ terms negligible under the conditions in Eqs. (3) and (6). This is an especially noteworthy result because all the conditions in Eq. (3) are perfectly satisfied as $a \rightarrow 0$, that is, as the radius of the charged sphere is allowed to approach the radius of a fundamental point-like particle (such as the electron).

\section{B. LAD equation of motion modified with transition forces}

There is, however, a problem with letting the radius $a$ of the charged sphere approach zero, namely the electrostatic mass $m_{\mathrm{es}}$ in Eq. (4) increases without bound as $a \rightarrow 0$; see (2). A fairly obvious way around this undesirable unbounded electrostatic mass is to assume, as Dirac did [37], that a fundamental particle like the electron is more complicated than the classical model of a charged insulator and simply renormalize the mass $m_{\mathrm{es}}$ to a fixed finite value $m$ equal to the measured rest mass of the fundamental particle (such as the electron) as $a$ is allowed to approach zero. Then the $\mathbf{O}(a)$ terms in Eq. (4) vanish and this modified LA equation of motion becomes equal to the modified LAD equation of motion

$$
\begin{aligned}
\mathbf{F}_{\mathrm{ext}}(t)+\mathbf{f}_{a}(t)= & m \frac{d}{d t}(\gamma \mathbf{u}) \\
& -\frac{e^{2}}{6 \pi \epsilon_{0} c^{3}}\left\{\frac{d}{d t}\left[\gamma^{2} \dot{\mathbf{u}}+\frac{\gamma^{4}}{c^{2}}(\mathbf{u} \cdot \dot{\mathbf{u}}) \mathbf{u}\right]\right. \\
& \left.-\frac{\gamma^{4}}{c^{2}}\left[|\dot{\mathbf{u}}|^{2}+\frac{\gamma^{2}}{c^{2}}(\mathbf{u} \cdot \dot{\mathbf{u}})^{2}\right] \mathbf{u}\right\},
\end{aligned}
$$

with renormalized finite mass $m$.

This modified LAD equation of motion in Eq. (7) is relativistically covariant, has the correct measured rest mass $m$ of the charged particle, contains no $\mathbf{O}(a)$ terms, and satisfies all the inequalities in Eq. (3) since $a$ has been allowed to approach zero. The transition forces $\mathbf{f}_{a}(t)=$ $\sum_{n=1}^{N} \mathbf{f}_{a n}(t)$ that eliminate the noncausality from the unmodified LAD equation of motion contain the delta function and the derivative of the delta function as $a \rightarrow 0$ $[28,29]$. The coefficients of these delta functions and their derivatives can be made unique by choosing the jumps in velocity across the transition intervals such that the momentum-energy radiated during each transition interval is equal to zero. These jumps in velocity are physically reasonable because they imply that the point charge moves across the transition interval to both minimize the radiated momentum-energy and keep its motion reversible across the transition interval where the externally applied force does no work on the charge as $a \rightarrow 0$. Also, these velocity jumps across the transition intervals are compatible with the inequalities in Eqs. (6) and (9) below, and are 
approximately equal to the changes in velocity produced by the preacceleration or predeceleration in the equation of motion unmodified by the transition forces.

Despite the attractive features of the causal modified LAD equation of motion in Eq. (7), it still requires one restrictive condition for its validity, namely the inequality corresponding to Eq. (5) that ensures a non-negative radiated energy across each transition interval so that the equation of motion conserves momentum energy. It can be shown $[28,29]$ that this inequality remains a required condition because with the renormalized mass $m$ of the particle in Eq. (7) as $a \rightarrow 0$, the factor $a / c$ in Eq. (5) is replaced by the finite time constant

$$
\tau_{e}=\frac{e^{2}}{6 \pi \epsilon_{0} m c^{3}}=6.27 \times 10^{-24} \mathrm{~s},
$$

for an electron and $m_{\mathrm{es}}$ in Eq. (5) is replaced with the finite value of the measured rest mass $m$. Specifically, we have

$$
\frac{\tau_{e}\left|\Delta \mathbf{F}_{\mathrm{ext}}^{n}\right|}{m c} \ll 1,
$$

in the rest frame as the one remaining condition required to ensure the validity of the causal modified LAD equation of motion in Eq. (7) (besides the transition intervals being separated in time by an amount on the order of $\tau_{e}$ or greater). Also, Eq. (9) ensures that Eq. (6) is satisfied for the rest-frame velocity jumps across transition intervals chosen to minimize the momentum energy radiated across the transition intervals.

There is some justification, even in classical physics, for renormalizing the mass $m_{\mathrm{es}}+m_{\text {ins }}$ to a finite value $m$ as $a \rightarrow 0\left(m_{\mathrm{es}} \rightarrow \infty\right)$ to obtain the equation of motion of a point charge because $m_{\text {ins }}$ can include gravitational and other attractive formation energies. Thus, as $a \rightarrow 0$ it is conceivable, even classically, that $m_{\text {ins }} \rightarrow-\infty$ and that $\lim _{a \rightarrow 0}\left(m_{\mathrm{es}}+m_{\mathrm{ins}}\right)=m$, the measured rest mass of the charged particle. It is, therefore, disconcerting that for the causal modified LAD equation of motion in Eq. (7), the restriction in Eq. (9) on the magnitude of the change in the externally applied force across a transition interval is needed to ensure that the causal modified LAD equation of motion satisfies conservation of momentum energy (by keeping the value of the energy radiated during the transition intervals non-negative).

For the extended charged sphere, the corresponding condition in Eq. (5) is inconsequential because it is implied by the condition in the second inequality of (3a) needed to make negligible the $\mathbf{O}(a)$ terms in the proper-frame equation of motion. This condition in (3a) merely says that the $\mathbf{O}(a)$ terms may not be negligible if the speed of the charged sphere changes by an appreciable fraction of the speed of light in the time it takes light to cross the sphere.
As $a \rightarrow 0$ and the mass is renormalized to a finite value $m$, the conditions in Eq. (3) are all satisfied and one might expect that the resulting causal modified LAD equation of motion (7) would remain valid regardless of the magnitude of the changes in the externally applied force. This is not always the case, however, if the change in external force is large enough across a transition interval to disobey (9) because then it has been shown in Refs. [28,29] that the value of the energy radiated during the transition intervals can become negative and the momentum energy of the mass-renormalized charged particle is not conserved. Renormalization of the causal modified LA classical equation of motion of the extended charge in Eq. (4), an equation consistent with momentum-energy conservation, changes the scale factor between the Newtonian acceleration term and the radiation reaction term such that the renormalized causal modified LAD equation of motion in Eq. (7) does not always satisfy momentum-energy conservation if the change in the externally applied force is large enough across a transition interval.

For an electron encountering an abrupt change $\Delta E$ in the external rest-frame electric field, the inequality in Eq. (9) is satisfied unless $\Delta E k m c /\left(e \tau_{e}\right)=6 \pi \epsilon_{0} m^{2} c^{4} / e^{3}=$ $2.7 \times 10^{20} \mathrm{~V} / \mathrm{m}$, an enormously high-electric field that is about 200 times larger than the Schwinger critical electric field (see Eq. (87)) that can produce electron-positron pairs from the quantum vacuum. ${ }^{4}$ Thus Eq. (9) is an academic restriction that is violated only if the externally applied LF is so large that quantum effects could dominate and the classical equation of motion may no longer apply. Nonetheless, a classical equation of motion of a massrenormalized point charge that is both causal and conserves momentum-energy no matter how large the change in the external force across a transition interval does not result by simply equating the sum of the point-charge radiation reaction force and the externally applied force to the relativistic Newtonian acceleration force (measured rest mass times relativistic acceleration) and inserting the necessary delta-function transition forces at the nonanalytic

\footnotetext{
${ }^{4}$ If a magnetic field $\mathbf{B}$ is also present, the Schwinger critical field required for electron-positron pair production depends on the Maxwellian field invariants $E^{2}-c^{2} B^{2}$ and $c \mathbf{E} \cdot \mathbf{B}$. In a plane wave, both these invariants are zero and there is no pair production [38]. However, it is interesting that renormalization of a fully causal classical equation of motion has revealed a limitation on the magnitude of the allowed change in the externally applied fields across transition intervals that is consistent with the limitation on the magnitude of the fields that avoids quantum vacuum effects. Moreover, the rest-frame inequality in (9) is satisfied whenever the rest-frame inequality in (102) is satisfied for quantum recoil effects to be negligible for an electron in a counterpropagating laser beam, for which (9) can be rewritten as $\omega_{0} \tau_{e} a_{0} \ll 1$ say $\lesssim 1 / 10$ with $\left|\Delta \mathbf{F}_{\text {ext }}^{n}\right|=e E_{0}$ (assuming a rectangular-envelope plane-wave pulse with an amplitude that increases abruptly from zero to $E_{0}$ and decreases abruptly from $E_{0}$ to zero) and $a_{0}$ defined in (28b).
} 
points in time of the external force to obtain (7). A causal classical equation of motion of a mass-renormalized point charge that also conserves momentum-energy with a nonnegative radiated energy during the transition intervals for arbitrarily large changes in the external force across the transition intervals, if it exists, must involve a more complicated combining of the Newtonian and radiation reaction forces with the externally applied force than just a simple summation. A fully satisfactory classical equation of motion of a point charge with finite mass (as opposed to an extended charge with unrenormalized mass) does not presently exist.

It seems prudent, therefore, to simply accept Eq. (7) as the classical causal equation of motion of a massrenormalized point charge under the restriction in Eq. (9) on the magnitude of the change in the externally applied force across the transition intervals, or to tolerate the slight noncausality in the original LAD equation of motion given by Eq. (7) without the transition forces $\mathbf{f}_{a}(t)$. Practically, the preacceleration/deceleration of the LAD equation of motion unmodified by the transition forces is generally too small to have a significant bearing on the solution. Also, power series solutions like the LL approximate solution to the LAD equation of motion do not display the preacceleration/deceleration that exists in the exact solution to the unmodified LAD equation of motion.

\section{Unmodified LAD equation of motion}

For most practical problems, including the problem of a laser beam incident upon a relativistic electron, the noncausal preaccelerations and predecelerations in the exact solution of the unmodified LAD equation of motion have a negligible effect on the solution except during insignificantly short time intervals on the order of $\tau_{e}$ when the external force is first applied and when it is terminated. Thus, for practical purposes, we need not retain the transition forces in Eq. (7) nor the restriction in Eq. (9) on the change in the externally applied force across the transition intervals. We need consider only the LAD equation of motion unmodified by the transition forces, namely

$$
\begin{aligned}
\mathbf{F}_{\mathrm{ext}}(t)= & m \frac{d}{d t}(\gamma \mathbf{u})-m \tau_{e}\left\{\frac{d}{d t}\left[\gamma^{2} \dot{\mathbf{u}}+\frac{\gamma^{4}}{c^{2}}(\mathbf{u} \cdot \dot{\mathbf{u}}) \mathbf{u}\right]\right. \\
& \left.-\frac{\gamma^{4}}{c^{2}}\left[|\dot{\mathbf{u}}|^{2}+\frac{\gamma^{2}}{c^{2}}(\mathbf{u} \cdot \dot{\mathbf{u}})^{2}\right] \mathbf{u}\right\} .
\end{aligned}
$$

The dot product with this force equation gives the corresponding power equation of motion

$$
\begin{aligned}
\mathbf{F}_{\mathrm{ext}} \cdot \mathbf{u}= & m c^{2} \frac{d \gamma}{d t} \\
& -m \tau_{e}\left\{\frac{d}{d t}\left(\gamma^{4} \mathbf{u} \cdot \dot{\mathbf{u}}\right)-\gamma^{4}\left[|\dot{\mathbf{u}}|^{2}+\frac{\gamma^{2}}{c^{2}}(\mathbf{u} \cdot \dot{\mathbf{u}})^{2}\right]\right\}
\end{aligned}
$$

with its reversible kinetic power, reversible Schott radiation-reaction power, and the irreversible radiated power.

It is noteworthy that when the method of successive substitutions is applied to obtain a solution to the LAD equation of motion in Eq. (10a), such as the LL approximate solution given in the Landau-Lifshitz approximate solution section, this approximate solution is causal and, depending on the value of the externally applied force at the nonanalytic points of time, may exhibit delta-function transition forces when it is substituted back into the LAD equation (10a), thus recovering the form of the modified causal LAD equation of motion in Ref. (7). Of course, this LL approximate solution with the recovered transition forces, although causal, will not in general satisfy the differential equation in Eqs. (7) or (10a), nor will the recovered transition forces equal the correct transition forces in Ref. (7).

\section{THE LANDAU-LIFSHITZ APPROXIMATE SOLUTION}

Few exact solutions have been found to the LAD nonlinear differential equation of motion in Eq. (10a) [39]. Thus, a common approach to obtain a solution to the equation of motion is to assume the radiation-reaction terms on the right-hand side of (10a) are small compared to the first term (the relativistic momentum term) and derive an approximate solution for $d(\gamma \mathbf{u}) / d t$ by means of the method of successive substitutions. This was originally done by Landau and Lifshitz [[40] Secs. 75-76] to obtain an approximate solution with the first-order substitution of the LF $\mathbf{F}_{\mathrm{ext}}=e(\mathbf{E}+\mathbf{u} \times \mathbf{B})$.

An easy way to derive the LL approximate solution from the equations in Eq. (10) is to first substitute $\gamma^{4}\left[|\dot{\mathbf{u}}|^{2}+\gamma^{2}(\mathbf{u} \cdot \dot{\mathbf{u}})^{2} / c^{2}\right]$ from (10b) into (10a) to recast this equation in the form [[28] Chap. 7]

$$
\gamma \dot{\mathbf{u}}=\frac{1}{m}\left[\mathbf{F}_{\mathrm{ext}}-\left(\mathbf{F}_{\mathrm{ext}} \cdot \mathbf{u}\right) \frac{\mathbf{u}}{c^{2}}\right]+\frac{\tau_{e}}{\gamma} \frac{d}{d t}\left(\gamma^{3} \dot{\mathbf{u}}\right) .
$$

Assuming the radiation-reaction term in Eq. (11) is small compared to the external force terms, substitute

$$
\gamma \dot{\mathbf{u}}=\frac{1}{m}\left[\mathbf{F}_{\mathrm{ext}}-\left(\mathbf{F}_{\mathrm{ext}} \cdot \mathbf{u}\right) \frac{\mathbf{u}}{c^{2}}\right]
$$

into the right-hand side of Eq. (10a) and use the corresponding approximation $d \gamma / d t \approx\left(\mathbf{F}_{\mathrm{ext}} \cdot \mathbf{u}\right) /\left(m c^{2}\right)$ gleaned from (10b) to obtain the first-order successive-substitution solution to Eq. (10a) as

$$
\begin{aligned}
m \frac{d(\gamma \mathbf{u})}{d t} \approx & \mathbf{F}_{\mathrm{ext}}+\tau_{e}\left[\frac{\left(\mathbf{u} \cdot \mathbf{F}_{\mathrm{ext}}\right) \mathbf{F}_{\mathrm{ext}}}{m c^{2}}+\gamma \frac{d \mathbf{F}_{\mathrm{ext}}}{d t}\right. \\
& \left.-\frac{\gamma^{2}}{m c^{2}}\left(\left|\mathbf{F}_{\mathrm{ext}}\right|^{2}-\frac{\left(\mathbf{u} \cdot \mathbf{F}_{\mathrm{ext}}\right)^{2}}{c^{2}}\right) \mathbf{u}\right] .
\end{aligned}
$$


With $\mathbf{F}_{\mathrm{ext}}=e(\mathbf{E}+\mathbf{u} \times \mathbf{B})$ such that $d \mathbf{F}_{\mathrm{ext}} / d t=e(d \mathbf{E} / d t+$ $\mathbf{u} \times d \mathbf{B} / d t+\dot{\mathbf{u}} \times \mathbf{B}$ ), and $\dot{\mathbf{u}}$ inserted from Eq. (12), one obtains the LL approximate solution as [[28] Eq. (8.132a)]

$$
\begin{aligned}
m \frac{d(\gamma \mathbf{u})}{d t} \approx & e(\mathbf{E}+\mathbf{u} \times \mathbf{B})+e \tau_{e} \gamma\left[\frac{d \mathbf{E}}{d t}+\mathbf{u} \times \frac{d \mathbf{B}}{d t}\right] \\
& +\frac{e^{2} \tau_{e}}{m c}\left[\frac{(\mathbf{u} \cdot \mathbf{E})}{c} \mathbf{E}+c(\mathbf{E}+\mathbf{u} \times \mathbf{B}) \times \mathbf{B}\right] \\
& +\frac{e^{2} \tau_{e} \gamma^{2}}{m c^{2}}\left[\frac{(\mathbf{u} \cdot \mathbf{E})^{2}}{c^{2}}-|\mathbf{E}+\mathbf{u} \times \mathbf{B}|^{2}\right] \mathbf{u} .
\end{aligned}
$$

Taking the dot product of $\mathbf{u}$ with Eq. (14a) produces the corresponding power equation of motion [[28] Eq. (8.132b)]

$$
\begin{aligned}
m c^{2} \frac{d \gamma}{d t} \approx & e \mathbf{u} \cdot \mathbf{E}+e \tau_{e} \gamma \mathbf{u} \cdot \frac{d \mathbf{E}}{d t} \\
& +\frac{e^{2} \tau_{e}}{m}\left[|\mathbf{E}|^{2}+(\mathbf{u} \times \mathbf{B}) \cdot \mathbf{E}\right] \\
& +\frac{e^{2} \tau_{e} \gamma^{2}}{m}\left[\frac{(\mathbf{u} \cdot \mathbf{E})^{2}}{c^{2}}-|\mathbf{E}+\mathbf{u} \times \mathbf{B}|^{2}\right] .
\end{aligned}
$$

We note that $d \mathbf{E} / d t$ denotes the total time derivative, so that

$$
\frac{d \mathbf{E}(\mathbf{r}, t)}{d t}=\frac{\partial \mathbf{E}(\mathbf{r}, t)}{\partial t}+\mathbf{u}(t) \cdot \nabla \mathbf{E}(\mathbf{r}, t) .
$$

The first term on the right-hand side of Eqs. (14a) and (14b) is the Lorentz force and power, respectively, the last term (multiplied by $\gamma^{2}$ ) on the right-hand side of Eqs. (14a) and $(14 b)$ is the negative of the approximate radiation force and power, and the middle terms on the right-hand side of Eqs. (14a) and (14b) are the negative of the Schott force and power. The radiated power (negative of last term) in Eq. (14b) is manifestly non-negative and irreversible. The Schott force and power (negative of middle terms) in Eqs. (14a) and (14b) are not perfect differentials and thus not manifestly reversible. This imperfect reversibility of the LL Schott force-power is a shortcoming of the LL approximate solution.

\section{A. Accuracy of the LL approximation obtained from the small-velocity LAD and LL solutions}

For $u / c \ll 1$, the LAD solution in Eq. (10a) reduces to

$$
m \dot{\mathbf{u}}=e(\mathbf{E}+\mathbf{u} \times \mathbf{B})+m \tau_{e}\left(\ddot{\mathbf{u}}-\mathbf{u}|\dot{\mathbf{u}}|^{2} / c^{2}\right),
$$

or as $u / c \rightarrow 0$

$$
m \dot{\mathbf{u}}=e \mathbf{E}+m \tau_{e} \ddot{\mathbf{u}} .
$$

Similarly, for $u / c \ll 1$, the LL approximate solution in Eq. (14a) reduces to

$$
\begin{aligned}
m \dot{\mathbf{u}} \approx & e(\mathbf{E}+\mathbf{u} \times \mathbf{B})+e \tau_{e}(\dot{\mathbf{E}}+\mathbf{u} \times \dot{\mathbf{B}}) \\
& +\frac{e^{2} \tau_{e}}{m c^{2}}\left[\mathbf{u} \cdot\left(\mathbf{E} \mathbf{E}+c^{2} \mathbf{B} \mathbf{B}\right)-\mathbf{u}\left(|\mathbf{E}|^{2}+c^{2}|\mathbf{B}|^{2}\right)\right. \\
& \left.+c^{2} \mathbf{E} \times \mathbf{B}\right] .
\end{aligned}
$$

For plane waves with frequency $\omega_{0}$ in the rest frame of the charge, the time derivatives produce a factor of $\omega_{0}$ and (17) further reduces to

$$
\begin{aligned}
m \dot{\mathbf{u}} \approx & e(\mathbf{E}+\mathbf{u} \times \mathbf{B}) \\
& +\frac{e^{2} \tau_{e}}{m c^{2}}\left[\mathbf{u} \cdot\left(\mathbf{E} \mathbf{E}+c^{2} \mathbf{B} \mathbf{B}\right)-2 \mathbf{u}|\mathbf{E}|^{2}+c^{2} \mathbf{E} \times \mathbf{B}\right],
\end{aligned}
$$

under the condition

$$
\omega_{0} \tau_{e} \ll 1
$$

With $u / c \rightarrow 0$, the LL approximation for $m \dot{\mathbf{u}}$ in Eq. (18) becomes

$$
m \dot{\mathbf{u}} \approx e \mathbf{E}+\frac{e^{2} \tau_{e}}{m} \mathbf{E} \times \mathbf{B} .
$$

Taking the time derivative of Eq. (18) and letting $u / c \rightarrow 0$ gives the corresponding LL approximation for $\ddot{\mathbf{u}}$

$$
\begin{aligned}
\ddot{\mathbf{u}} \approx & \frac{e}{m}(\dot{\mathbf{E}}+\dot{\mathbf{u}} \times \mathbf{B})+\frac{e^{2} \tau_{e}}{m^{2} c^{2}}\left[\dot{\mathbf{u}} \cdot\left(\mathbf{E} \mathbf{E}+c^{2} \mathbf{B B}\right)\right. \\
& \left.-2 \dot{\mathbf{u}}|\mathbf{E}|^{2}+c^{2} \frac{d}{d t}(\mathbf{E} \times \mathbf{B})\right]
\end{aligned}
$$

which becomes after substituting $\dot{\mathbf{u}}$ from Eqs. (20) into (21) and noting that $d(\mathbf{E} \times \mathbf{B}) / d t \sim \omega_{0} \mathbf{E} \times \mathbf{B}$ so that the condition in Eq. (19) eliminates this term

$$
\begin{aligned}
\ddot{\mathbf{u}} \approx & \frac{e}{m} \dot{\mathbf{E}}+\frac{e^{2}}{m^{2}} \mathbf{E} \times \mathbf{B} \\
& -\frac{e^{2} \tau_{e}}{m^{2} c^{2}}\left[2 \frac{e}{m}|\mathbf{E}|^{2} \mathbf{E}+2 \frac{e^{2} \tau_{e}}{m^{2}}|\mathbf{E}|^{2} \mathbf{E} \times \mathbf{B}\right] .
\end{aligned}
$$

Note that to get this correct LL rest-frame expression for $\ddot{\mathbf{u}}$, the small-velocity terms containing $\mathbf{u}$ had to be initially included in the LL equation (18) for $\dot{\mathbf{u}}$.

With this LL approximation for $\ddot{\mathbf{u}}$ in Eq. (22) inserted into the LAD equation of motion (16b), the LAD equation for $m \dot{\mathbf{u}}$ becomes [upon noting that $\dot{\mathbf{E}} \sim \omega_{0} \mathbf{E}$ and omitting this term because of Eq. (19)]

$$
\begin{aligned}
m \dot{\mathbf{u}}= & e \mathbf{E}+\frac{e^{2} \tau_{e}}{m} \mathbf{E} \times \mathbf{B} \\
& -\frac{e^{2} \tau_{e}^{2}}{m c^{2}}\left[2 \frac{e}{m}|\mathbf{E}|^{2} \mathbf{E}+2 \frac{e^{2} \tau_{e}}{m^{2}}|\mathbf{E}|^{2} \mathbf{E} \times \mathbf{B}\right]
\end{aligned}
$$


or

$$
m \dot{\mathbf{u}}=\left(e \mathbf{E}+\frac{e^{2} \tau_{e}}{m} \mathbf{E} \times \mathbf{B}\right)\left(1-2 \omega_{0}^{2} \tau_{e}^{2} a_{0}^{2}\right)
$$

where $a_{0}$ is the dimensionless laser-strength parameter defined below in Eq. (28b).

A comparison of Eqs. (20) and (24) reveals that for plane-wave excitations the LL approximation is an accurate solution to the LAD equation of motion if and only if, in addition to the inequality in Eq. (19)

$$
\omega_{0} \tau_{e} a_{0} \ll 1
$$

in the rest frame of the charge. The two rest-frame accuracy conditions, (19) and (25), for the validity of the LL approximation with plane-wave excitations can also be obtained using the simplified heuristic argument of Landau and Lifshitz [[40] Eqs. (75.11) and (75.12)]. The accuracy conditions in Eqs. (19) and (25), generalized to the laboratory frame of an electron in a counterpropagating plane wave, are given as Eqs. (80) and (83)-(84) in Sec. V below.

\section{RELATIVISTIC ELECTRON IN A COUNTERPROPAGATING PLANE WAVE}

The solution to the LL equation of motion will be obtained for an electron with an arbitrary initial velocity illuminated by a counterpropagating plane-wave pulse with an arbitrary intensity. A closed-form solution for the subsequent velocity of the electron can be found explicitly as a function of time for a general polarization of the plane wave (any combination of two orthogonal linear polarizations with an arbitrary phase difference). However, because the explicit expressions for the generalpolarization solution are quite lengthy, attention will be confined to the two most interesting and topical polarizations, namely linear and circular polarization. Since the LL equation, like the LAD equation, is nonlinear in velocity, two polarization solutions cannot be added together to get a third polarization solution. In particular, the solution for the circularly polarized plane wave is not equal to the sum of the solutions to two linearly polarized plane waves ninety degrees out of phase.

\section{A. Linearly polarized plane wave}

Consider an electron with initial speed $u_{0}$ (at $t=0$ and $z=0$ ) moving in the $+z$ direction oppositely to the direction of propagation $-z$ of a pulsed, linearly polarized plane wave having an electric-field $\mathbf{E}$ in the $+x$ direction and a magnetic-field $\mathbf{B}$ in the $-y$ direction. The fields of the linearly polarized incident plane wave can be written as

$$
\mathbf{E}=E(\omega t+k z) \cos (\omega t+k z) \hat{\mathbf{x}}=E(\xi) \cos \xi \hat{\mathbf{x}}
$$

$$
\mathbf{B}=-\frac{1}{c} E(\omega t+k z) \cos (\omega t+k z) \hat{\mathbf{y}}=-\frac{1}{c} E(\xi) \cos \xi \hat{\mathbf{y}},
$$

where $B=E / c, \omega=2 \pi f$ is the angular frequency $(f>0$ is the frequency), and $k=\omega / c=2 \pi / \lambda$ is the propagation constant with $c$ the free-space speed of light. The envelope of the pulse is given by $E(\omega t+k z)$. Following the notation in [17], we have let

$$
\xi=\omega t+k z
$$

so that

$$
\frac{d \xi}{d t}=\omega\left(1+u_{z} / c\right)
$$

Because the derivative in Eq. (26d) is greater than or equal to zero, the retarded time parameter $\xi$ is a monotonically increasing function of the real time $t$ and, vice versa, the real time $t$ is a monotonically increasing function of the retarded time parameter $\xi$. If a phase $\xi_{0}$ is added to the $\omega t+k z$ in Eqs. (26a) and (26b), then $\xi=\omega t+k z+$ $\xi_{0}$ in Eq. (26c). Since this generalization adds little to the physics, $\xi_{0}$ has been chosen equal to zero. The force exerted on the electron by the fields of the linearly polarized plane wave produces velocity changes in both the $x$ and $z$ directions, but not in the $y$ direction. Since the electron is moving through the plane wave, the longitudinal coordinate in Eq. (26) at the position of the moving electron is a function of time, that is, $z=z(t)$ with $u_{z}=d z / d t$. The plane-wave fields given in Eq. (26) constitute an exact solution to Maxwell's equations because there is no variation in the fields with $x$ and $y$, that is, no field variation transverse to the direction of propagation.

The $x$ and $z$ components of the force-momentum equation of the LL approximate solution (14a) to the LAD equation of motion can be written for the electron as

$$
\begin{aligned}
\frac{d\left(\gamma u_{x} / c\right)}{d \xi}= & -a_{0}\left[\mathcal{E} \cos \xi+\omega \tau_{e} \gamma\left(1+u_{z} / c\right) d(\mathcal{E} \cos \xi) / d \xi\right] \\
& -\omega \tau_{e} a_{0}^{2} \gamma\left(1+u_{z} / c\right)\left(\gamma u_{x} / c\right) \mathcal{E}^{2} \cos ^{2} \xi \\
\frac{d\left(\gamma u_{z} / c\right)}{d \xi}= & a_{0}\left[\mathcal{E} \cos \xi /\left(1+u_{z} / c\right)+\omega \tau_{e} \gamma d(\mathcal{E} \cos \xi) / d \xi\right] \\
& \cdot\left(u_{x} / c\right)-\omega \tau_{e} a_{0}^{2}\left[1+\left(1+u_{z} / c\right) \gamma^{2}\left(u_{z} / c\right)\right] \mathcal{E}^{2} \cos ^{2} \xi
\end{aligned}
$$

where

$$
E(\xi)=E_{0} \mathcal{E}(\xi)
$$

with $E_{0}$ equal to the maximum magnitude of the envelope of the pulse and 


$$
a_{0}=\frac{e E_{0}}{m c \omega}
$$

is the dimensionless laser-strength parameter. Here and throughout the rest of the paper $e$ denotes the positive magnitude of the charge of the electron, $m$ is the mass of the electron, $\tau_{e}$ is given in Eq. (8), and $u^{2}=u_{x}^{2}+u_{z}^{2}$. Taking the dot product of the velocity vector $\mathbf{u}$ with the time rate of change of momentum to get $u_{x} d\left(\gamma u_{x}\right) / d \xi+u_{z} d\left(\gamma u_{z}\right) / d \xi=$ $c^{2} d \gamma / d \xi$, we find from Eq. (27) that

$$
\begin{aligned}
\frac{d \gamma}{d \xi}= & -a_{0}\left[\mathcal{E} \cos \xi /\left(1+u_{z} / c\right)+\omega \tau_{e} \gamma d(\mathcal{E} \cos \xi) / d \xi\right]\left(u_{x} / c\right) \\
& +\omega \tau_{e} a_{0}^{2}\left[1-\left(1+u_{z} / c\right) \gamma^{2}\right] \mathcal{E}^{2} \cos ^{2} \xi
\end{aligned}
$$

which can also be obtained directly from the power Eq. (14b) of the LL approximate solution to the LAD equation of motion. The $\cos \xi$ terms on the right-hand sides of Eqs. (27) and (29) are the Lorentz force-power delivered directly to the electron and the other terms are the LL approximation to the time rate of change of momentum energy caused by the radiation reaction. We want to solve the differential equations in Eqs. (27)-(29) for $u_{x}, u_{z}$, and $\gamma$ as functions of $\xi$ and then find $t$ as a function of $\xi$ in order to obtain explicit closedform expressions for $u_{x}, u_{z}$, and $\gamma$ as functions of time $t$.

\section{Solution for $\gamma\left(1+u_{z} / c\right)$}

A differential equation that is immediately solvable results by adding the two Eqs. (27b) and (29) to get

$$
\frac{d\left[\gamma\left(1+u_{z} / c\right)\right]}{d \xi}=-\omega \tau_{e} a_{0}^{2} \gamma^{2}\left(1+u_{z} / c\right)^{2} \mathcal{E}^{2} \cos ^{2} \xi .
$$

Dividing through by $\left(1+u_{z} / c\right)^{2}$ and integrating from 0 to $\xi$, one readily determines the solution as

$$
\gamma\left(1+u_{z} / c\right)=\frac{\gamma_{z 0}}{1+a_{0}^{2} \gamma_{z 0} \omega \tau_{e} \mathcal{F}(\xi)},
$$

where $\gamma_{z 0}=\gamma_{0}\left(1+u_{z 0} / c\right)$ with the initial values of $\gamma$ and $u_{z}$ given by $\gamma_{0}=\gamma(0)$ and $u_{z 0}=u_{z}(0)$. The integral function $\mathcal{F}(\xi)$ is found from Eq. (30) as

$$
\mathcal{F}(\xi)=\int_{0}^{\xi} \mathcal{E}^{2}\left(\xi^{\prime}\right) \cos ^{2} \xi^{\prime} d \xi^{\prime}
$$

If the longitudinal velocity $u_{z}$ in Eq. (31) is approximated by $c$ so that $\gamma_{z 0}=2 \gamma_{0}$ and $\mathcal{F}(\xi)$ is approximated by $\xi / 2 \approx \omega t$ for $\mathcal{E}=1$, then the solution for $\gamma(t)$ equals the one obtained previously by Vranic et al. [13] and Thomas et al. [41]

$$
\gamma(t)=\frac{\gamma_{0}}{1+2 a_{0}^{2} \gamma_{0} \omega^{2} \tau_{e} t},
$$

which, besides its approximate nature, has the problem that $\gamma(t) \rightarrow 0$ as $t \rightarrow \infty$.

The longitudinal velocity function $\gamma u_{z} / c$ is obtained directly from Eq. (31) as

$$
\frac{\gamma u_{z}}{c}=\frac{\gamma_{z 0}}{\mathcal{G}(\xi)}-\gamma
$$

with

$$
\mathcal{G}(\xi)=1+a_{0}^{2} \gamma_{z 0} \omega \tau_{e} \mathcal{F}(\xi) .
$$

Next, it is shown that the closed-form expression for $\gamma\left(1+u_{z} / c\right)$ in Eq. (31) enables one to find a closed-form expression for $\gamma u_{x} / c$.

\section{Solution for $\gamma u_{x} / c$}

Insertion of $\gamma\left(1+u_{z} / c\right)$ from Eqs. (31) into (27a) yields a first-order linear differential equation for $\gamma u_{x} / c$

$$
\begin{aligned}
& \frac{d\left(\gamma u_{x} / c\right)}{d \xi}+\omega \tau_{e} a_{0}^{2} \gamma_{z 0} \frac{\mathcal{E}^{2} \cos ^{2} \xi}{\mathcal{G}}\left(\gamma u_{x} / c\right) \\
& =-a_{0}\left[\mathcal{E} \cos \xi+\omega \tau_{e} \gamma_{z 0} \frac{d(\mathcal{E} \cos \xi) / d \xi}{\mathcal{G}}\right] .
\end{aligned}
$$

Rewriting this equation in the more compact form

$$
\frac{d\left(\gamma u_{x} / c\right)}{d \xi}+P(\xi)\left(\gamma u_{x} / c\right)=Q(\xi)
$$

allows the standard linear first-order differentialequation solution in terms of an integrating factor to be expressed as [42]

$$
\frac{d\left(\gamma u_{x} / c\right)}{d \xi}=\frac{1}{e^{I(\xi)}} \int_{0}^{\xi} Q\left(\xi^{\prime}\right) e^{I\left(\xi^{\prime}\right)} d \xi^{\prime},
$$

with

$$
I(\xi)=\int_{0}^{\xi} P\left(\xi^{\prime}\right) d \xi^{\prime}
$$

Substitution of $P(\xi)$ and $Q(\xi)$ from Eqs. (36) into (37) yields

$$
\frac{\gamma u_{x}}{c}=-a_{0} \frac{\mathcal{H}(\xi)}{\mathcal{G}(\xi)}
$$

where

$$
\begin{gathered}
\mathcal{H}(\xi)=\mathcal{S}(\xi)+\omega \tau_{e} \gamma_{z 0}\left[\mathcal{E}(\xi) \cos \xi-\mathcal{E}(0)+a_{0}^{2} \mathcal{C}(\xi)\right], \\
\mathcal{S}(\xi)=\int_{0}^{\xi} \mathcal{E}\left(\xi^{\prime}\right) \cos \xi^{\prime} d \xi^{\prime}
\end{gathered}
$$




$$
\mathcal{C}(\xi)=\int_{0}^{\xi} \mathcal{E}\left(\xi^{\prime}\right) \mathcal{F}\left(\xi^{\prime}\right) \cos \xi^{\prime} d \xi^{\prime}
$$

Of course, one still needs $\gamma$ to evaluate $u_{x} / c$ in Eq. (38).

\section{Solution for the relativistic factor $\gamma$, the longitudinal velocity $u_{z} / c$, and the transverse velocity $u_{x} / c$}

To find an explicit expression for the relativistic factor $\gamma$ and longitudinal velocity $u_{z} / c$, given $\gamma u_{z} / c$ in Eq. (34) and $\gamma u_{x} / c$ in Eq. (38), we use the following form of the definition of $\gamma$ in terms of the velocities

$$
\gamma^{2}=1+\left(\gamma u_{x} / c\right)^{2}+\left(\gamma u_{z} / c\right)^{2},
$$

which yields $\gamma$ from Refs. (34) and (38) as

$$
\gamma \mathcal{G}(\xi)=\frac{1}{2 \gamma_{z 0}}\left[\gamma_{z 0}^{2}+\mathcal{G}^{2}(\xi)+a_{0}^{2} \mathcal{H}^{2}(\xi)\right] .
$$

Having obtained $\gamma \mathcal{G}(\xi)$ (and thus $\gamma$ ), we can write the longitudinal velocity $u_{z} / c$ from Eq. (34) as

$$
\frac{u_{z}}{c}=\frac{\gamma_{z 0}}{\gamma \mathcal{G}(\xi)}-1,
$$

and the transverse velocity $u_{x} / c$ from Eq. (38) as

$$
\frac{u_{x}}{c}=-a_{0} \frac{\mathcal{H}(\xi)}{\gamma \mathcal{G}(\xi)} .
$$

For envelope functions $\mathcal{E}(\xi)$ equal to the sum of trigonometric and polynomial functions of $\xi$ and their products, the integrals in Eqs. (32) and (39) needed to determine $\mathcal{G}(\xi)$ and $\mathcal{H}(\xi)$ in Eqs. (41)-(43) can be evaluated in closed form.

\section{Determination of the time $\boldsymbol{t}$ in terms of $\xi$}

The solutions for $\gamma, u_{z} / c$, and $u_{x} / c$ in Eqs. (41), (42), and (43), respectively, are given in terms of the parameter $\xi=\omega(t+z / c)$ and, because $z(t)=\int_{0}^{t} u_{z}\left(t^{\prime}\right) d t^{\prime}$, it would appear well-nigh impossible to solve the equation $\xi=$ $\omega[t+z(t) / c]$ explicitly for $t$ in terms of $\xi$. Fortunately, this is not the case. If $z$ can be found explicitly as a function of $\xi$, that is, if one can determine $z[t(\xi)]$ explicitly, then $t$ is given as

$$
t=\xi / \omega-z[t(\xi)] / c .
$$

To find $z[t(\xi)] / c$ in terms of $\xi$, note that it can be expressed as

$$
\begin{aligned}
\frac{z[t(\xi)]}{c} & =\frac{1}{c} \int_{0}^{\xi} \frac{d z\left[t\left(\xi^{\prime}\right)\right]}{d \xi^{\prime}} d \xi^{\prime}=\frac{1}{c} \int_{0}^{\xi} \frac{d z(t)}{d t} \frac{d t}{d \xi^{\prime}} d \xi^{\prime} \\
& =\int_{0}^{\xi} \frac{u_{z}\left[t\left(\xi^{\prime}\right)\right] / c}{\omega\left(1+u_{z}\left[t\left(\xi^{\prime}\right)\right] / c\right)} d \xi^{\prime}
\end{aligned}
$$

and, thus, from Eq. (44)

$$
\begin{aligned}
t & =\frac{1}{\omega} \int_{0}^{\xi}\left[1-\frac{u_{z}\left[t\left(\xi^{\prime}\right)\right] / c}{\left(1+u_{z}\left[t\left(\xi^{\prime}\right)\right] / c\right)}\right] d \xi^{\prime} \\
& =\frac{1}{\omega} \int_{0}^{\xi} \frac{d \xi^{\prime}}{\left(1+u_{z}\left[t\left(\xi^{\prime}\right)\right] / c\right)} .
\end{aligned}
$$

Insertion of $\left(1+u_{z}\left[t\left(\xi^{\prime}\right)\right] / c\right)$ from Eq. (42) produces the convenient expression for $t$ in terms of $\xi$ as

$$
t=\frac{1}{\omega \gamma_{z 0}} \int_{0}^{\xi} \gamma\left(\xi^{\prime}\right) \mathcal{G}\left(\xi^{\prime}\right) d \xi^{\prime}
$$

where $\gamma(\xi) \mathcal{G}(\xi)$ is given in Eq. (41). For envelope functions $\mathcal{E}(\xi)$ equal to the sum of trigonometric and polynomial functions of $\xi$ and their products, the integral in Eq. (47) can be evaluated in closed form. Alternatively, $t$ can be found from a straightforward computer summation of $\Delta t=$ $\gamma\left(\xi^{\prime}\right) \mathcal{G}\left(\xi^{\prime}\right) \Delta \xi^{\prime}$ from $\xi^{\prime}=0$ to $\xi$ for the chosen differential increments $\Delta \xi^{\prime}$.

\section{Uniform plane wave}

For a uniform plane wave, the envelope function $\mathcal{E}(\xi)=$ 1 and the integrals for $\mathcal{F}(\xi), \mathcal{S}(\xi)$, and $\mathcal{C}(\xi)$ in Eqs. (32), $(39 b)$, and (39c) evaluate to

$$
\begin{gathered}
\mathcal{F}(\xi)=\frac{1}{2}\left(\xi+\frac{1}{2} \sin 2 \xi\right) \\
\mathcal{S}(\xi)=\sin \xi \\
\mathcal{C}(\xi)=\frac{1}{2} \xi \sin \xi-\frac{1}{6} \cos ^{3} \xi+\frac{1}{2} \cos \xi-\frac{1}{3}
\end{gathered}
$$

When these expressions are substituted into Eqs. (35) and (39a) to obtain $\mathcal{G}(\xi)$ and $\mathcal{H}(\xi)$, the relativistic factor and velocities in Eqs. (41)-(43) agree with those derived by Hadad et al. [[17] Eq. (A1)] (if the typographical errors are corrected in equation (A1) of Ref. [17] as personally communicated by Y. Hadad). Also, the directions of $u_{x}$ and $u_{z}$ in Ref. [17] are opposite those herein because the initial longitudinal velocity of the electron in Ref. [17] is chosen in the $-z$ direction. Although it is not necessary to evaluate the integral for the time $t$ in Eq. (47) explicitly (since it can be computed as a simple summation), for the sake of completeness or if faster computational speed is desired, the explicit closed-form expression for $t$ in terms of $\xi$ is 


$$
\begin{aligned}
\omega \gamma_{z 0} t= & \left(\gamma_{0}+\frac{a_{0}^{2}}{4 \gamma_{z 0}}\right) \xi-\frac{a_{0}^{2}}{8 \gamma_{z 0}} \sin 2 \xi+\omega \tau_{e}\left[a_{0}^{2}\left(\frac{1}{4} \xi^{2}-\frac{3}{8} \cos 2 \xi+\cos \xi-\frac{5}{8}\right)\right. \\
& \left.+a_{0}^{4}\left(\frac{1}{8} \xi^{2}-\frac{1}{8} \xi \sin 2 \xi+\frac{1}{24} \cos ^{4} \xi-\frac{3}{16} \cos 2 \xi+\frac{1}{3} \cos \xi-\frac{3}{16}\right)\right] \\
& +\omega^{2} \tau_{e}^{2} \gamma_{z 0} a_{0}^{2}\left\{\left[\frac{3}{4} \xi+\frac{1}{8} \sin 2 \xi-\sin \xi+a_{0}^{2}\left(\frac{1}{24} \xi^{3}+\frac{103}{192} \xi-\frac{3}{16} \xi \cos 2 \xi+\frac{1}{2} \xi \cos \xi-\frac{1}{18} \sin ^{3} \xi-\frac{7}{768} \sin 4 \xi\right.\right.\right. \\
& \left.\left.+\frac{17}{96} \sin 2 \xi-\frac{7}{6} \sin \xi\right)\right]+\frac{a_{0}^{4}}{2}\left[\frac{1}{24} \xi^{3}+\frac{1}{6} \xi-\frac{1}{16} \xi^{2} \sin 2 \xi-\frac{3}{16} \xi \cos 2 \xi+\frac{1}{24} \xi \cos ^{4} \xi+\frac{1}{3} \xi \cos \xi\right. \\
& \left.\left.+\frac{1}{216} \sin \xi \cos ^{3} \xi\left(\cos ^{2} \xi+\frac{5}{4}\right)-\frac{1}{27} \sin ^{3} \xi-\frac{5}{768} \sin 4 \xi+\frac{125}{1152} \sin 2 \xi-\frac{5}{9} \sin \xi\right]\right\}
\end{aligned}
$$

It should be noted that in Ref. [17], the time $t$ is not found as a function of $\xi$, as is done in Eqs. (47) or (49). Instead, the proper time $\tau$ is found as a function of $\xi$ so that $\gamma, u_{x} / c$, and $u_{z} / c$ can be expressed numerically as functions of $\tau$. Then $t$ is apparently determined as a function of $\tau$ by numerically evaluating the integral $t=\int_{0}^{\tau} \gamma\left(\tau^{\prime}\right) d \tau^{\prime}$.

Since $\gamma, u_{x} / c$, and $u_{z} / c$ are plotted vs time $t$ in Ref. [17], these plots will not be repeated here. The power supplied by the externally applied force to the electron, the power radiated, the kinetic power, and the Schott power are not plotted in Ref. [17]; thus these powers, normalized to $m c^{2}$ and given in units of $\mathrm{s}^{-1}$, are shown in Figs. 1-3. These powers, found from Eq. (29) with $d \xi$ inserted from Eq. (26d), can be expressed respectively as

$$
\begin{gathered}
P_{\mathrm{ext}}=-\omega a_{0}\left(u_{x} / c\right) \mathcal{E} \cos \xi \\
P_{\mathrm{rad}}=\omega^{2} \tau_{e} a_{0}^{2}\left(1+u_{z} / c\right)^{2} \gamma^{2} \mathcal{E}^{2} \cos ^{2} \xi \\
P_{\mathrm{kin}}=\frac{d \gamma}{d t}=\omega\left(1+u_{z} / c\right) \frac{d \gamma}{d \xi}
\end{gathered}
$$

and

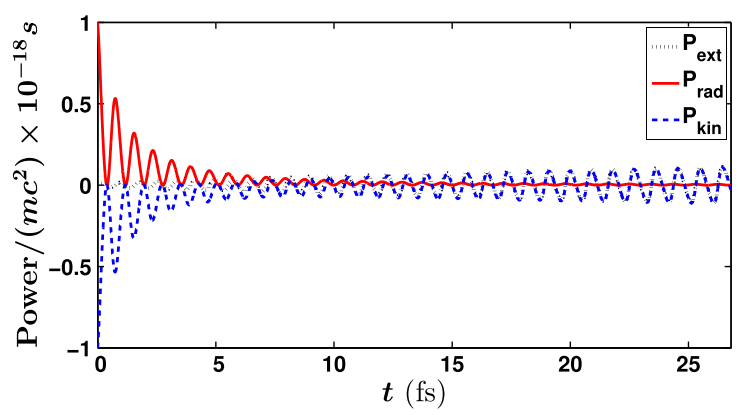

FIG. 1. $\quad P_{\text {ext }}, P_{\text {rad }}$, and $P_{\text {kin }}$ plotted vs time $t$ for a uniform linearly polarized laser with angular frequency $\omega=2 \times 10^{15} \mathrm{~s}^{-1}$ and strength $a_{0}=100(\mathcal{E}=1)$, and an electron with initial relativistic factor $\gamma_{0}=1000$.

$$
\begin{aligned}
P_{\mathrm{Sch}}= & \omega^{2} \tau_{e} a_{0}\left(1+u_{z} / c\right)\left[\gamma\left(u_{x} / c\right) \frac{d}{d \xi}(\mathcal{E} \cos \xi)\right. \\
& \left.-a_{0} \mathcal{E}^{2} \cos ^{2} \xi\right]
\end{aligned}
$$

The laser-strength parameter $a_{0}$ is chosen equal to 100 , the initial relativistic factor $\gamma_{0}$ is chosen equal to 1000 and the angular frequency $\omega$ of the laser equals $2 \times 10^{15} \mathrm{~s}^{-1}$ in order to conform to the choices for these parameters in Ref. [17]. For the uniform plane wave, we have $\mathcal{E}=1$. The plots cover the time interval from 0 to 26.8 fs (several

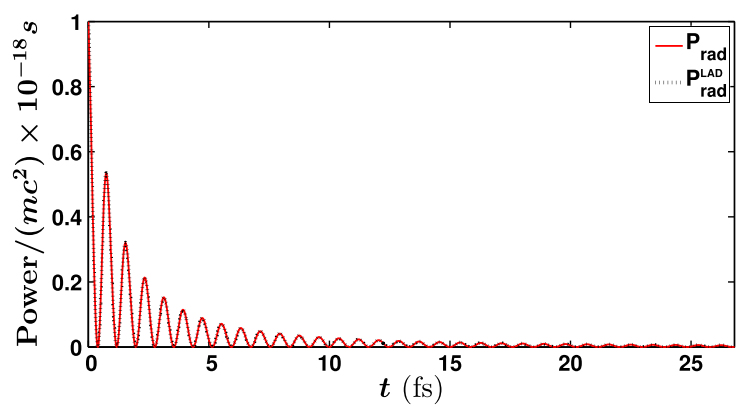

FIG. 2. Comparison of $P_{\mathrm{rad}}(t)$ and $P_{\mathrm{rad}}^{\mathrm{LAD}}(t)$ for the uniform linearly polarized plane wave with parameters given in Fig. 1.

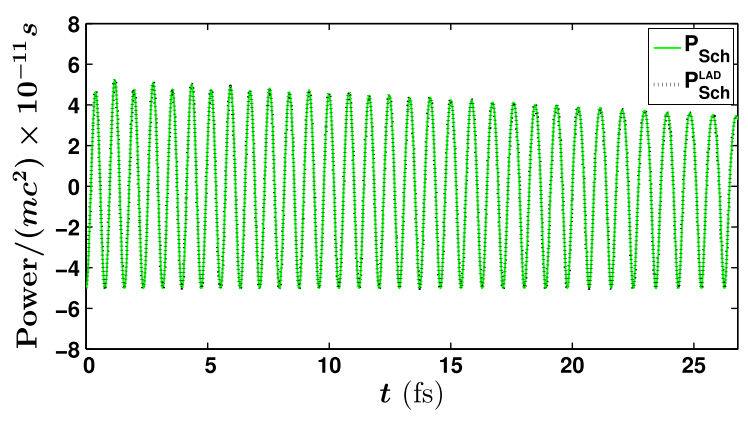

FIG. 3. Comparison of $P_{\mathrm{Sch}}(t)$ and $P_{\mathrm{Sch}}^{\mathrm{LAD}}(t)$ for the uniform linearly polarized plane wave with parameters given in Fig. 1. 
periods of the laser beam), again to conform to the time interval chosen in Ref. [17].

One can see in Fig. 1 that the power $\left(P_{\text {ext }}\right)$ supplied by the external electric-field force of the laser beam is practically equal to the sum of the kinetic power $\left(P_{\text {kin }}\right)$ and the radiated power $\left(P_{\text {rad }}\right)$ because the Schott power shown in Fig. 3 is more than five orders of magnitude smaller than the sum of these other powers. The radiated power can also be computed by substituting the values of $\mathbf{u} / c$ and $\dot{\mathbf{u}} / c$ determined from the LL solution into the radiated power in the LAD power equation of motion (10b) (normalized to $m c^{2}$ ), that is

$$
P_{\mathrm{rad}}^{\mathrm{LAD}}=\tau_{e} \gamma^{4}\left[\left|\frac{\dot{\mathbf{u}}}{c}\right|^{2}+\frac{\gamma^{2}}{c^{2}}\left(\frac{\mathbf{u} \cdot \dot{\mathbf{u}}}{c^{2}}\right)^{2}\right] .
$$

As Fig. 2 shows, the difference between $P_{\text {rad }}$ and $P_{\text {rad }}^{\mathrm{LAD}}$ is not detectable within the linewidth of the curves, thereby indicating the high accuracy of the LL solution for the given frequency and strength of the laser beam, and for the initial velocity (relativistic factor) of the electron. Equations (81) and (82) below show that the fractional error $\eta_{\text {rad }}$ in the LL approximate radiated power for uniform linear polarization is given by

$\eta_{\mathrm{rad}}=\frac{P_{\mathrm{rad}}^{\mathrm{LAD}}-P_{\mathrm{rad}}}{P_{\mathrm{rad}}}=\omega^{2} \tau_{e}^{2} a_{0}^{2}\left(1+u_{z} / c\right)^{2} \gamma^{2} \cos ^{2} \xi$

which, for the present case depicted in Fig. 2, has a maximum value of about $6 \times 10^{-6}$ at $\gamma=\gamma_{0}=1000$. The error expression for uniform circular polarization is identical to that given in Eq. (52) but without the $\cos ^{2} \xi$ factor.

The Schott power can also be computed by substituting the values of $\mathbf{u} / c$ and $\dot{\mathbf{u}} / c$ determined from the LL solution into the Schott power in the power LAD equation of motion (10b). Rewriting $\gamma^{4} \mathbf{u} \cdot \dot{\mathbf{u}} / c^{2}$ as $\gamma d \gamma / d t$ and approximating $d \gamma / d t$ by $\mathbf{F}_{\mathrm{ext}} \cdot \mathbf{u} /\left(m c^{2}\right)$, we have

$$
\begin{aligned}
P_{\mathrm{Sch}}^{\mathrm{LAD}} & =\omega \tau_{e} a_{0} \frac{d}{d t}\left[\gamma\left(u_{x} / c\right) \mathcal{E} \cos \xi\right] \\
& =\omega^{2} \tau_{e} a_{0}\left(1+u_{z} / c\right) \frac{d}{d \xi}\left[\gamma\left(u_{x} / c\right) \mathcal{E} \cos \xi\right] .
\end{aligned}
$$

The plots of $P_{\mathrm{Sch}}(t)$ and $P_{\mathrm{Sch}}^{\mathrm{LAD}}(t)$ in Fig. 3 reveal that there is no noticeable difference between the two evaluations of the Schott power. This is especially noteworthy because not only is the Schott power several orders of magnitude below the other powers but the LL Schott power $\left(P_{\mathrm{Sch}}\right)$ is not perfectly reversible (as pointed out in Sec. III), whereas the LAD Schott power $\left(P_{\mathrm{Sch}}^{\mathrm{LAD}}\right)$ is a reversible perfect time differential. This implies that for this example of the relativistic electron in a counterpropagating high-intensity laser beam, the approximate LL irreversible Schott power is closely approximated by the LAD reversible Schott power.

\section{Sinusoidal envelope}

A more realistic laser-beam pulse begins continuously from a value of zero and ends continuously with a value of zero, such as the envelope function

$$
\mathcal{E}(\xi)=\sin (\alpha \xi), \quad 0 \leq \xi \leq \pi / \alpha,
$$

where $\alpha$ is a constant. For $\alpha \ll 1$, the envelope contains many cycles of the laser-beam frequency. With this sinusoidal envelope function in Eq. (54), the integrals for $\mathcal{F}(\xi), \mathcal{S}(\xi)$, and $\mathcal{C}(\xi)$ in Eqs. (32), (39b), and (39c) evaluate to

$$
\begin{aligned}
\mathcal{F}(\xi)= & \frac{1}{2} \sin ^{2}(\alpha \xi)\left(\xi+\frac{1}{2} \sin 2 \xi\right) \\
& +\frac{1}{4}\left(\xi \cos (2 \alpha \xi)-\frac{\sin (2 \alpha \xi)}{2 \alpha}\right) \\
& -\frac{\alpha}{16}\left[\frac{\sin (2(1-\alpha) \xi)}{1-\alpha}-\frac{\sin (2(1+\alpha) \xi)}{1+\alpha}\right], \\
\mathcal{S}(\xi)= & \frac{1}{2}\left[\frac{1-\cos ((1+\alpha) \xi)}{1+\alpha}-\frac{1-\cos ((1-\alpha) \xi)}{1-\alpha}\right], \\
\mathcal{C}(\xi)= & \mathcal{S}_{0}(\xi) \mathcal{F}(\xi) \\
& -\frac{1}{1-\alpha^{2}}\left[\frac{\alpha}{2}\left(\mathcal{C}_{\alpha}-\mathcal{C}_{3 \alpha}\right)-\frac{1}{3} \sin ^{3}(\alpha \xi) \cos ^{3} \xi\right],
\end{aligned}
$$

where

$$
\mathcal{S}_{0}(\xi)=\mathcal{S}(\xi)+\frac{\alpha}{1-\alpha^{2}},
$$

$$
\begin{aligned}
\mathcal{C}_{\alpha}(\xi)= & \frac{1}{3+\alpha}\left\{\cos ^{3} \xi \sin (\alpha \xi)\right. \\
& -\frac{3}{1+\alpha}\left[\cos ^{2} \xi \sin ((1-\alpha) \xi)-\frac{\sin ((3-\alpha) \xi)}{3-\alpha}\right. \\
& \left.\left.-\frac{\sin ((1-\alpha) \xi)}{1-\alpha}\right]\right\} .
\end{aligned}
$$

The integral for time in Eq. (47) can be evaluated in closed form but the expressions are even more tedious than those given in Eq. (49) for the uniform plane wave and they are not necessary since the integral in Eq. (47) can be accurately and rapidly evaluated by a simple summation in computational software such as MATLAB.

The relativistic factor $\gamma$, the longitudinal velocity $u_{z} / c$, and the transverse velocity $u_{x} / c$ are plotted vs time $t$ in Figs. 4, 5, and 6 for the sinusoidal envelope $\mathcal{E}=\sin (\alpha \xi)$ with $\alpha=0.029715$. This value of $\alpha$ is chosen to give an envelope pulse width of $26.8 \mathrm{fs}$, the length of the uniformplane-wave pulse chosen above and in Ref. [17]. A comparison of these plots with the corresponding plots given in Ref. [17] for the uniform plane wave (rectangular 
pulse) reveals that the sinusoidal envelope, which begins and ends continuously with a value of zero, produces significant changes in $\gamma, u_{z} / c$, and $u_{x} / c$. The relativistic factor $\gamma$ in Fig. 4 has a zero slope at $t=0$ and decreases much more slowly with time than the corresponding uniform-plane-wave $\gamma$ plotted in Ref. [17]. Over the 26.8 fs time interval, $\gamma$ remains much greater than unity $(u / c \approx 1)$ for both uniform and sinusoidal linearly

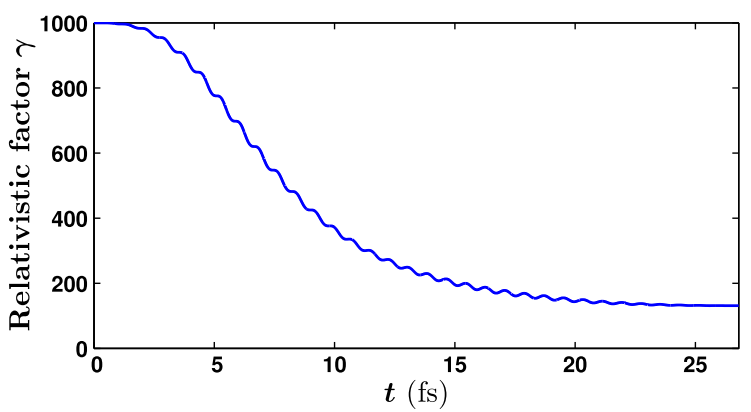

FIG. 4. Relativistic factor $\gamma$ plotted vs time $t$ for a sinusoidalenvelope linearly polarized laser pulse with angular frequency $\omega=2 \times 10^{15} \mathrm{~s}^{-1}$, maximum strength $a_{0}=100$, and a pulsewidth equal to 26.8 fs. The electron has an initial relativistic factor $\gamma_{0}=1000$.

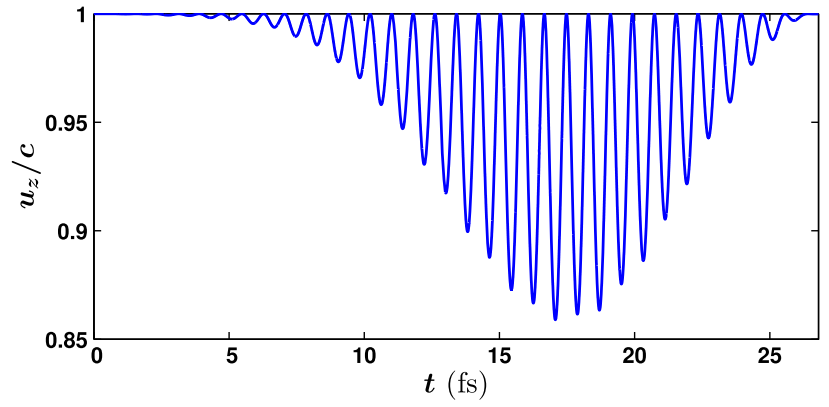

FIG. 5. Longitudinal velocity $u_{z} / c$ plotted vs time $t$ for a sinusoidal-envelope linearly polarized laser pulse with parameters given in Fig. 4.

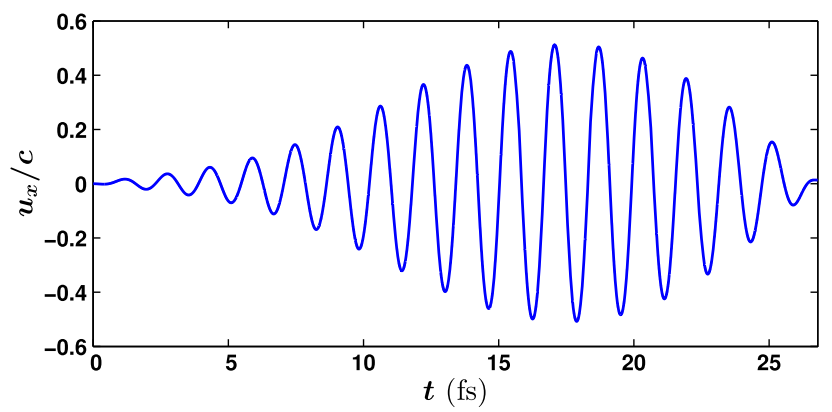

FIG. 6. Transverse velocity $u_{x} / c$ plotted vs time $t$ for a sinusoidal-envelope linearly polarized laser pulse with parameters given in Fig. 4. polarized plane waves. The envelope of the transverse velocity $u_{x} / c$ in Fig. 6 for the sinusoidal laser-pulse envelope not only begins at a value of zero but also ends by decreasing slowly to a value close to zero. During the same time, the envelope of the longitudinal velocity $u_{z} / c$ in Fig. 5 ends by increasing slowly from appreciably less than the speed of light to very close to the speed of light $\left(u_{z} / c \approx 0.99988131\right.$ at $\left.t=26.8 \mathrm{fs}\right)$.

The power supplied by the externally applied force to the electron, the power radiated, the kinetic power, and the Schott power for the sinusoidal envelope are plotted in Figs. 7-9. As with the uniform plane wave (rectangular

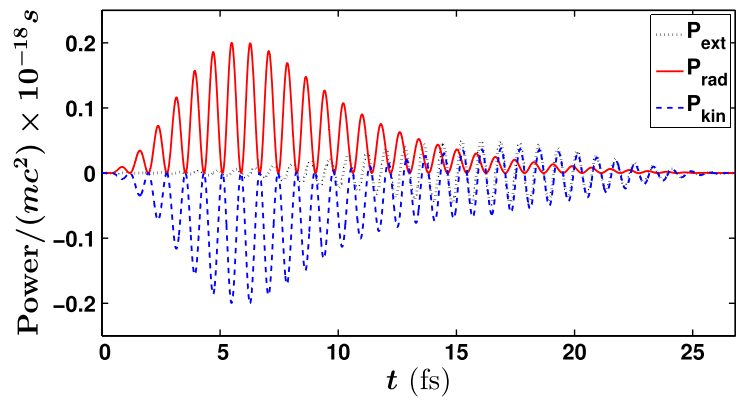

FIG. 7. $\quad P_{\text {ext }}, P_{\text {rad }}$, and $P_{\text {kin }}$ plotted vs time $t$ for a sinusoidalenvelope linearly polarized laser with parameters given in Fig. 4.

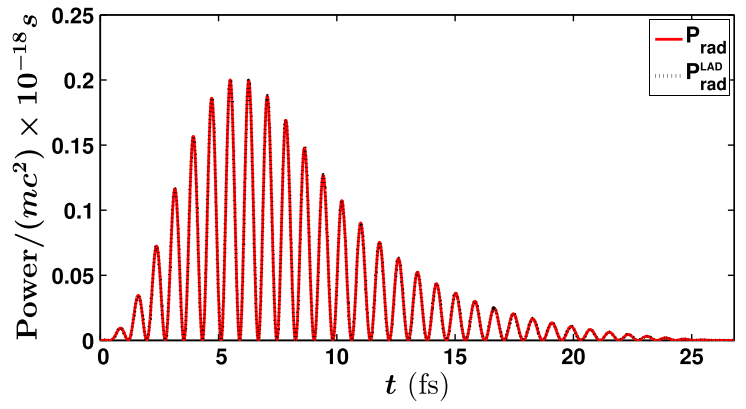

FIG. 8. Comparison of $P_{\text {rad }}(t)$ and $P_{\text {rad }}^{\mathrm{LAD}}(t)$ for the sinusoidalenvelope linearly polarized plane wave with parameters given in Fig. 4.

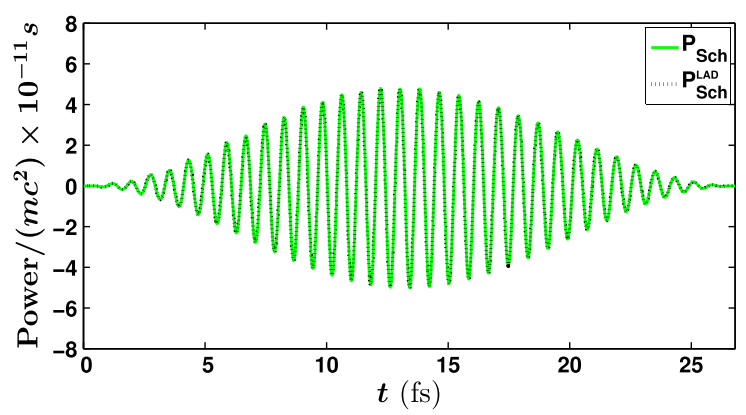

FIG. 9. Comparison of $P_{\mathrm{Sch}}(t)$ and $P_{\mathrm{Sch}}^{\mathrm{LAD}}(t)$ for the sinusoidalenvelope linearly polarized plane wave with parameters given in Fig. 4. 
pulse), Fig. 7 shows that the power $\left(P_{\text {ext }}\right)$ supplied by the external electric-field force of the laser beam is practically equal to the sum of the kinetic power $\left(P_{\text {kin }}\right)$ and the radiated power $\left(P_{\text {rad }}\right)$ because the Schott power shown in Fig. 9 is so much smaller than the sum of these other powers. Also, as Fig. 8 shows, the difference between $P_{\text {rad }}$ and $P_{\text {rad }}^{\mathrm{LAD}}$ is not detectable within the linewidth of the curves, again indicating the high accuracy of the LL solution; see Eq. (52). The curves for $P_{\mathrm{Sch}}(t)$ and $P_{\mathrm{Sch}}^{\mathrm{LAD}}(t)$ in Fig. 9 again exhibit no noticeable difference between these two evaluations of the Schott power. The most obvious difference between the power curves for the uniform and sinusoidal-envelope pulses is the vanishing of the large radiated power near the beginning of the sinusoidal-envelope pulse.

Surprisingly, the total energy radiated (time integral of power radiated over the pulse) in the case of the sinusoidalenvelope pulse is not much less than the total energy radiated in the case of the uniform plane wave (rectangular-envelope pulse); specifically the total radiated energy normalized to $m c^{2}$ is found to be $W_{\text {rad }} \simeq W_{\text {rad }}^{\mathrm{LAD}} \approx 938$ (uniform pulse), 872 (sinusoidal pulse). The total energy supplied by the plane-wave fields of the laser beam is about twelve times higher for the uniform rectangular pulse than for the sinusoidal-envelope pulse; specifically the total supplied energy normalized to $m c^{2}$ is found to be $W_{\text {ext }} \approx 36.5$ (uniformpulse), 3.1 (sinusoidal pulse). In either case, most of the radiated energy is taken from the change in kinetic energy of the electron rather than the energy supplied by the plane-wave fields.

The total scattering cross section of the electron as determined by the total radiated energy during the $26.8 \mathrm{fs}$ divided by the total incident energy per unit area in the plane wave illuminating the electron during the $26.8 \mathrm{fs}$ is equal to $1.85 \times 10^{-23} \mathrm{~m}^{2}$ and $3.45 \times 10^{-23} \mathrm{~m}^{2}$ for the uniform and sinusoidal-envelope pulses, respectively. Interestingly, these total scattering cross sections are much closer to $\pi \lambda_{c}^{2}=1.85 \times 10^{-23} \mathrm{~m}^{2}$, the area of a circle with a radius equal to the Compton wavelength $\lambda_{c}$, than to $\pi r_{e}^{2}=2.5 \times 10^{-29} \mathrm{~m}^{2}$, the area of a circle with a radius equal to the classical radius $r_{e}$ of the electron.

\section{B. Circularly polarized plane wave}

Next consider the case of a circularly polarized laser beam [43], that is, an electron with initial speed $u_{0}$ (at $t=0$ and $z=0$ ) moving in the $+z$ direction oppositely to the direction of propagation $-z$ of a pulsed, circularly polarized plane wave. The fields of the circularly polarized incident plane wave can be written as

$$
\mathbf{E}=E_{0} \mathcal{E}(\xi)[\sin \xi \hat{\mathbf{x}}+\cos \xi \hat{\mathbf{y}}],
$$

$\mathbf{B}=-\frac{E_{0}}{c} \mathcal{E}(\xi)[\sin \xi \hat{\mathbf{y}}-\cos \xi \hat{\mathbf{x}}], \quad B_{0}=E_{0} / c$, where for this circular polarization there is a $y$ component of the velocity as well as $x$ and $z$ components.

The $x, y$, and $z$ components of the force-momentum equation of the LL approximate solution (14a) to the LAD equation of motion can be written for the electron as

$$
\begin{aligned}
\frac{d\left(\gamma u_{x} / c\right)}{d \xi}= & -a_{0}\left[\mathcal{E} \sin \xi+\omega \tau_{e} \gamma\left(1+u_{z} / c\right) d(\mathcal{E} \sin \xi) / d \xi\right] \\
& -\omega \tau_{e} a_{0}^{2} \gamma\left(1+u_{z} / c\right)\left(\gamma u_{x} / c\right) \mathcal{E}^{2} \\
\frac{d\left(\gamma u_{y} / c\right)}{d \xi}= & -a_{0}\left[\mathcal{E} \cos \xi+\omega \tau_{e} \gamma\left(1+u_{z} / c\right) d(\mathcal{E} \cos \xi) / d \xi\right] \\
& -\omega \tau_{e} a_{0}^{2} \gamma\left(1+u_{z} / c\right)\left(\gamma u_{y} / c\right) \mathcal{E}^{2}, \\
\frac{d\left(\gamma u_{z} / c\right)}{d \xi}= & a_{0}\left[\mathcal{E}\left(\frac{u_{x}}{c} \sin \xi+\frac{u_{y}}{c} \cos \xi\right) /\left(1+u_{z} / c\right)\right. \\
+ & \left.+\omega \tau_{e} \gamma\left(\frac{u_{x}}{c} d(\mathcal{E} \sin \xi) / d \xi+\frac{u_{y}}{c} d(\mathcal{E} \cos \xi) / d \xi\right)\right] \\
& -\omega \tau_{e} a_{0}^{2}\left[1+\left(1+u_{z} / c\right) \gamma^{2}\left(u_{z} / c\right)\right] \mathcal{E}^{2} .
\end{aligned}
$$

Taking the dot product of the velocity vector $\mathbf{u}$ with the time rate of change of momentum to get $u_{x} d\left(\gamma u_{x}\right) / d \xi+u_{y} d\left(\gamma u_{y}\right) / d \xi+u_{z} d\left(\gamma u_{z}\right) / d \xi=c^{2} d \gamma / d \xi$, we find from Eq. (58) that

$$
\begin{aligned}
\frac{d \gamma}{d \xi}= & -a_{0}\left[\mathcal{E}\left(\frac{u_{x}}{c} \sin \xi+\frac{u_{y}}{c} \cos \xi\right) /\left(1+u_{z} / c\right)\right. \\
& \left.+\omega \tau_{e} \gamma\left(\frac{u_{x}}{c} d(\mathcal{E} \sin \xi) / d \xi+\frac{u_{y}}{c} d(\mathcal{E} \cos \xi) / d \xi\right)\right] \\
& +\omega \tau_{e} a_{0}^{2}\left[1-\left(1+u_{z} / c\right) \gamma^{2}\right] \mathcal{E}^{2},
\end{aligned}
$$

which can also be obtained directly from the power equation (14b) of the LL approximate solution to the LAD equation of motion. We want to solve the differential equations in Eqs. (58)-(59) for $u_{x}, u_{y}, u_{z}$, and $\gamma$ as functions of $\xi$ and then find $t$ as a function of $\xi$ in order to obtain explicit closed-form expressions for $u_{x}, u_{y}, u_{z}$, and $\gamma$ as functions of time $t$.

\section{Solution for $\gamma\left(1+u_{z} / c\right)$ : Circular polarization}

A differential equation that is immediately solvable results by adding the two Eqs. (58c) and (59) to get

$$
\frac{d\left[\gamma\left(1+u_{z} / c\right)\right]}{d \xi}=-\omega \tau_{e} a_{0}^{2} \gamma^{2}\left(1+u_{z} / c\right)^{2} \mathcal{E}^{2} .
$$

Dividing through by $\left(1+u_{z} / c\right)^{2}$ and integrating from 0 to $\xi$, one readily determines the solution as

$$
\gamma\left(1+u_{z} / c\right)=\frac{\gamma_{z 0}}{1+a_{0}^{2} \gamma_{z 0} \omega \tau_{e} \mathcal{F}_{c}(\xi)},
$$


where $\gamma_{z 0}=\gamma_{0}\left(1+u_{z 0} / c\right)$ with the initial values of $\gamma$ and $u_{z}$ given by $\gamma_{0}=\gamma(0)$ and $u_{z 0}=u_{z}(0)$. The integral function $\mathcal{F}_{c}(\xi)$ is found from Eq. (60) as

$$
\mathcal{F}_{c}(\xi)=\int_{0}^{\xi} \mathcal{E}^{2}\left(\xi^{\prime}\right) d \xi^{\prime} .
$$

The longitudinal velocity function $\gamma u_{z} / c$ is obtained directly from Eq. (61) as

$$
\frac{\gamma u_{z}}{c}=\frac{\gamma_{z 0}}{\mathcal{G}_{c}(\xi)}-\gamma
$$

with

$$
\mathcal{G}_{c}(\xi)=1+a_{0}^{2} \gamma_{z 0} \omega \tau_{e} \mathcal{F}_{c}(\xi) .
$$

The closed-form expression for $\gamma\left(1+u_{z} / c\right)$ in Eq. (61) enables one to find closed-form expressions for $\gamma u_{x} / c$ and $\gamma u_{y} / c$.

\section{Solution for $\gamma u_{x} / c$ and $\gamma u_{y} / c$ : Circular polarization}

Insertion of $\gamma\left(1+u_{z} / c\right)$ from Eqs. (61) into (58a) yields a first-order linear differential equation for $\gamma u_{x} / c$

$$
\begin{aligned}
& \frac{d\left(\gamma u_{x} / c\right)}{d \xi}+\omega \tau_{e} a_{0}^{2} \gamma_{z 0} \frac{\mathcal{E}^{2}}{\mathcal{G}_{c}}\left(\gamma u_{x} / c\right) \\
& =-a_{0}\left[\mathcal{E} \sin \xi+\omega \tau_{e} \gamma_{z 0} \frac{d(\mathcal{E} \sin \xi) / d \xi}{\mathcal{G}_{c}}\right] .
\end{aligned}
$$

Rewriting this equation in the more compact form

$$
\frac{d\left(\gamma u_{x} / c\right)}{d \xi}+P_{c}(\xi)\left(\gamma u_{x} / c\right)=Q_{c}(\xi)
$$

allows the solution to this standard linear first-order differential equation to be expressed as [42]

$$
\frac{\gamma u_{x}}{c}=-a_{0} \frac{\mathcal{H}_{x}(\xi)}{\mathcal{G}_{c}(\xi)}
$$

where

$$
\begin{gathered}
\mathcal{H}_{x}(\xi)=\mathcal{S}_{x}(\xi)+\omega \tau_{e} \gamma_{z 0}\left[\mathcal{E}(\xi) \sin \xi+a_{0}^{2} \mathcal{C}_{x}(\xi)\right], \\
\mathcal{S}_{x}(\xi)=\int_{0}^{\xi} \mathcal{E}\left(\xi^{\prime}\right) \sin \xi^{\prime} d \xi^{\prime}, \quad \text { and } \\
\mathcal{C}_{x}(\xi)=\int_{0}^{\xi} \mathcal{E}\left(\xi^{\prime}\right) \mathcal{F}_{c}\left(\xi^{\prime}\right) \sin \xi^{\prime} d \xi^{\prime}
\end{gathered}
$$

Similarly, insertion of $\gamma\left(1+u_{z} / c\right)$ from Eqs. (61) into (58b) yields a first-order linear differential equation for $\gamma u_{y} / c$, which solves to give

$$
\frac{\gamma u_{y}}{c}=-a_{0} \frac{\mathcal{H}_{y}(\xi)}{\mathcal{G}_{c}(\xi)}
$$

where

$$
\mathcal{H}_{y}(\xi)=\mathcal{S}_{y}(\xi)+\omega \tau_{e} \gamma_{z 0}\left[\mathcal{E}(\xi) \cos \xi-\mathcal{E}(0)+a_{0}^{2} \mathcal{C}_{y}(\xi)\right],
$$

$$
\begin{aligned}
& \mathcal{S}_{y}(\xi)=\int_{0}^{\xi} \mathcal{E}\left(\xi^{\prime}\right) \cos \xi^{\prime} d \xi^{\prime}, \quad \text { and } \\
& \mathcal{C}_{y}(\xi)=\int_{0}^{\xi} \mathcal{E}\left(\xi^{\prime}\right) \mathcal{F}_{c}\left(\xi^{\prime}\right) \cos \xi^{\prime} d \xi^{\prime} .
\end{aligned}
$$

Of course, one still needs $\gamma$ to evaluate $u_{x} / c$ and $u_{y} / c$ in Eqs. (66) and (68).

\section{Solution for the relativistic factor $\gamma$, the longitudinal velocity $u_{z} / c$, and the transverse velocities $u_{x} / c$ and $u_{y} / c$ : Circular polarization}

To find an explicit expression for the relativistic factor $\gamma$ and longitudinal velocity $u_{z} / c$, given $\gamma u_{z} / c$ in Eq. (63) and $\gamma u_{x} / c$ and $\gamma u_{y} / c$ in Eqs. (66) and (68), we use the following form of the definition of $\gamma$ in terms of the velocities

$$
\gamma^{2}=1+\left(\gamma u_{x} / c\right)^{2}+\left(\gamma u_{x} / c\right)^{2}+\left(\gamma u_{z} / c\right)^{2},
$$

which yields $\gamma$ from Eqs. (63), (66), and (68) as

$$
\gamma \mathcal{G}_{c}(\xi)=\frac{1}{2 \gamma_{z 0}}\left[\gamma_{z 0}^{2}+\mathcal{G}_{c}^{2}(\xi)+a_{0}^{2}\left(\mathcal{H}_{x}^{2}(\xi)+\mathcal{H}_{y}^{2}(\xi)\right)\right] .
$$

Having obtained $\gamma \mathcal{G}_{c}(\xi)$ (and thus $\gamma$ ), we can write the longitudinal velocity $u_{z} / c$ from Eq. (63) as

$$
\frac{u_{z}}{c}=\frac{\gamma_{z 0}}{\gamma \mathcal{G}_{c}(\xi)}-1
$$

and the transverse velocities $u_{x} / c$ and $u_{y} / c$ from Eqs. (66) and (68) as

$$
\begin{gathered}
\frac{u_{x}}{c}=-a_{0} \frac{\mathcal{H}_{x}(\xi)}{\gamma \mathcal{G}_{c}(\xi)} \quad \text { and } \\
\frac{u_{y}}{c}=-a_{0} \frac{\mathcal{H}_{y}(\xi)}{\gamma \mathcal{G}_{c}(\xi)} .
\end{gathered}
$$

For envelope functions $\mathcal{E}(\xi)$ equal to the sum of trigonometric and polynomial functions of $\xi$ and their products, the integrals in Eqs. (62), (67) and (69) needed to determine $\mathcal{G}_{c}(\xi), \mathcal{H}_{x}(\xi)$, and $\mathcal{H}_{y}(\xi)$ in Eqs. (71)-(73) can be evaluated in closed form. 
The time $t$ can also be determined similarly in closed form in terms of $\xi$ from the relationship derived in Eq. (47), which becomes for the circularly polarized plane wave

$$
t=\frac{1}{\omega \gamma_{z 0}} \int_{0}^{\xi} \gamma\left(\xi^{\prime}\right) \mathcal{G}_{c}\left(\xi^{\prime}\right) d \xi^{\prime},
$$

where $\gamma(\xi) \mathcal{G}_{c}(\xi)$ is given in Eq. (71). Alternatively, $t$ can be found from a straightforward computer summation of $\Delta t=$ $\gamma\left(\xi^{\prime}\right) \mathcal{G}_{c}\left(\xi^{\prime}\right) \Delta \xi^{\prime}$ from $\xi^{\prime}=0$ to $\xi$ for the chosen differential increments $\Delta \xi^{\prime}$.

\section{Uniform plane wave: Circular polarization}

For a uniform circularly polarized plane wave, the envelope function $\mathcal{E}(\xi)=1$ and the integrals for $\mathcal{F}_{c}(\xi)$, $\mathcal{S}_{x}(\xi), \mathcal{C}_{x}(\xi), \mathcal{S}_{y}(\xi)$, and $\mathcal{C}_{y}(\xi)$ in Eqs. (62), (67b), (67c), $(69 \mathrm{~b})$, and $(69 \mathrm{c})$ evaluate to

$$
\begin{gathered}
\mathcal{F}_{c}(\xi)=\xi, \\
\mathcal{S}_{x}(\xi)=1-\cos \xi, \\
\mathcal{C}_{x}(\xi)=-\xi \cos \xi+\sin \xi, \\
\mathcal{S}_{y}(\xi)=\sin \xi, \quad \text { and } \\
\mathcal{C}_{y}(\xi)=\xi \sin \xi+\cos \xi-1 .
\end{gathered}
$$

When these expressions are substituted into Eqs. (64), (67a), and (69a) to obtain $\mathcal{G}_{c}(\xi), \mathcal{H}_{x}(\xi)$, and $\mathcal{H}_{y}(\xi)$, the relativistic factor and velocities in Eqs. (71)-(73) agree with those derived by Hadad et al. [[17] Eq. (A2)], except that the directions of $u_{y}$ and $u_{z}$ in Ref. [17] are opposite those herein. Although it is not necessary to evaluate the integral for the time $t$ in Eq. (74) explicitly (since it can be computed as a simple summation), for the sake of completeness or if faster computational speed is desired, the explicit closed-form expression for $t$ in terms of $\xi$ is

$$
\begin{aligned}
\omega \gamma_{z 0} t= & \gamma_{0} \xi+a_{0}^{2}(\xi-\sin \xi) / \gamma_{z 0}+\omega \tau_{e} a_{0}^{2}\left[\xi^{2} / 2\right. \\
& \left.+a_{0}^{2}\left(\xi^{2} / 2-\xi \sin \xi-\cos \xi+1\right)\right] \\
& +\omega^{2} \tau_{e}^{2} \gamma_{z 0}\left[a_{0}^{2}(\xi-\sin \xi)+a_{0}^{4}(2 \xi-3 \sin \xi+\xi \cos \xi\right. \\
& \left.\left.+\xi^{3} / 6\right)+a_{0}^{6}\left(\xi-2 \sin \xi+\xi \cos \xi+\xi^{3} / 6\right)\right] .
\end{aligned}
$$

Again it is noted that in Ref. [17] the proper time $\tau$ rather than the actual time $t$ is found as a function of $\xi$ so that $\gamma, u_{x} / c, u_{y} / c$, and $u_{z} / c$ can be expressed numerically as functions of $\tau$. Then $t$ is apparently determined as a function of $\tau$ by numerically evaluating the integral $t=\int_{0}^{\tau} \gamma\left(\tau^{\prime}\right) d \tau^{\prime}$.

Since $\gamma, u_{x} / c$, and $u_{z} / c$ are plotted vs time $t$ in Ref. [17], and the variation of $u_{y} / c$ is similar to that of $u_{x} / c$, these plots will not be repeated here. The power supplied by the

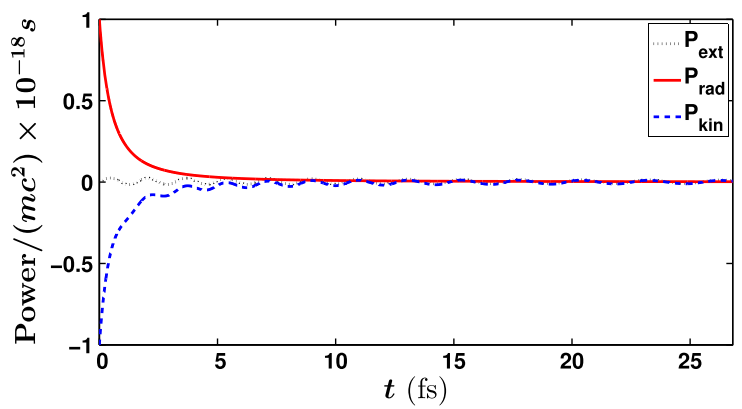

FIG. 10. $\quad P_{\text {ext }}, P_{\text {rad }}$, and $P_{\text {kin }}$ plotted vs time $t$ for a uniform circularly polarized laser with angular frequency $\omega=$ $2 \times 10^{15} \mathrm{~s}^{-1}$ and strength $a_{0}=100(\mathcal{E}=1)$, and an electron with initial relativistic factor $\gamma_{0}=1000$.

externally applied force to the electron, the power radiated, the kinetic power, and the Schott power are not plotted in Ref. [17]; thus these powers, normalized to $m c^{2}$ and given in units of $s^{-1}$, are plotted in Figs. 10 and 11. These powers, found from Eq. (59) with $d \xi$ inserted from (26d), can be expressed respectively as

$$
\begin{gathered}
P_{\text {ext }}=-\omega a_{0}\left(\frac{u_{x}}{c} \sin \xi+\frac{u_{y}}{c} \cos \xi\right) \mathcal{E}, \\
P_{\text {rad }}=\omega^{2} \tau_{e} a_{0}^{2}\left(1+u_{z} / c\right)^{2} \gamma^{2} \mathcal{E}^{2}, \\
P_{\text {kin }}=\frac{d \gamma}{d t}=\omega\left(1+u_{z} / c\right) \frac{d \gamma}{d \xi},
\end{gathered}
$$

and

$$
\begin{aligned}
P_{\mathrm{Sch}}= & \omega^{2} \tau_{e} a_{0}\left(1+u_{z} / c\right)\left[\gamma \left(\frac{u_{x}}{c} d(\mathcal{E} \sin \xi) / d \xi\right.\right. \\
& \left.\left.+\frac{u_{y}}{c} d(\mathcal{E} \cos \xi) / d \xi\right)-a_{0} \mathcal{E}^{2}\right]
\end{aligned}
$$

The laser-strength parameter $a_{0}$ is chosen equal to 100 , the initial relativistic factor $\gamma_{0}$ is chosen equal to 1000 and the angular frequency $\omega$ of the laser equals $2 \times 10^{15} \mathrm{~s}^{-1}$ in

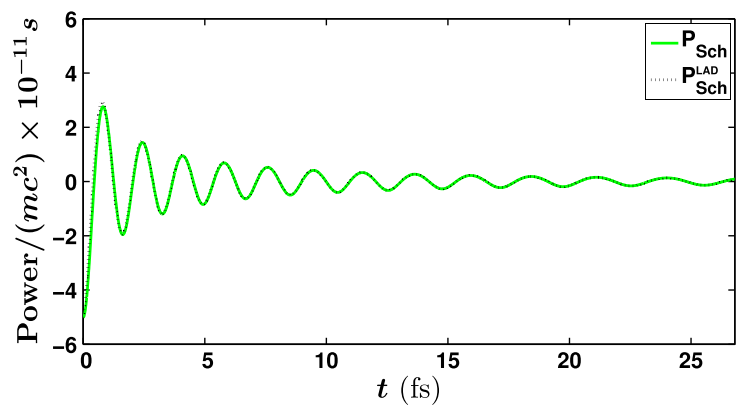

FIG. 11. $P_{\mathrm{Sch}}(t)$ and $P_{\mathrm{Sch}}^{\mathrm{LAD}}(t)$ for the uniform circularly polarized plane wave with parameters given in Fig. 10. 
order to conform to the choices for these parameters for the linearly polarized case above and in Ref. [17]. For the uniform plane wave, we have $\mathcal{E}=1$. The plots cover the time interval from 0 to 26.8 fs (several periods of the laser beam), again to conform to the time interval chosen above and in Ref. [17].

One can see in Fig. 10 that the power $\left(P_{\text {ext }}\right)$ supplied by the external electric-field force of the laser beam is practically equal to the sum of the kinetic power $\left(P_{\text {kin }}\right)$ and the radiated power $\left(P_{\text {rad }}\right)$ because the Schott power shown in Fig. 11 is more than five orders of magnitude smaller than the sum of these other powers. The radiated power can also be computed by substituting the values of $\mathbf{u} / c$ and $\dot{\mathbf{u}} / c$ determined from the LL solution into the radiated power in the LAD power equation of motion (10b); see Eq. (51). As in the linearly polarized case, the difference between $P_{\text {rad }}(t)$ and $P_{\mathrm{rad}}^{\mathrm{LAD}}(t)$ (not shown) is smaller than the linewidth of the $P_{\text {rad }}(t)$ curve in Fig. 10, thereby again indicating the high accuracy of the LL solution for the given frequency and strength of the laser beam, and for the initial velocity (relativistic factor) of the electron; see Eq. (52). Also, Fig. 11 shows that the Schott power $P_{\mathrm{Sch}}^{\mathrm{LAD}}(t)$ computed by substituting the values of $\mathbf{u} / c$ and $\dot{\mathbf{u}} / c$ determined from the LL solution into the Schott power in the power LAD equation of motion (10b) (Eq. (53) with $u_{x} \sin \xi+$ $u_{y} \cos \xi$ replacing $\left.u_{x} \cos \xi\right)$ is very close to $P_{\mathrm{Sch}}(t)$.

\section{Sinusoidal envelope: Circular polarization}

With the sinusoidal envelope function in Eq. (54) for the circular polarization, the integrals for $\mathcal{F}_{c}(\xi), \mathcal{S}_{x}(\xi), \mathcal{C}_{x}(\xi)$, $\mathcal{S}_{y}(\xi)$, and $\mathcal{C}_{y}(\xi)$ in Eqs. (62), (67b), (67c), (69b), and (69c) evaluate to

$$
\begin{gathered}
\mathcal{F}_{c}(\xi)=\frac{1}{2}\left(\xi-\frac{\sin (2 \alpha \xi}{2 \alpha}\right), \\
\mathcal{S}_{x}(\xi)=\frac{1}{2}\left[\frac{\sin [(1-\alpha) \xi]}{1-\alpha}-\frac{\sin [(1+\alpha) \xi]}{1+\alpha}\right], \\
\mathcal{C}_{x}(\xi)=\frac{1}{4}\left[\xi\left(\frac{\sin [(1-\alpha) \xi]}{1-\alpha}-\frac{\sin [(1+\alpha) \xi]}{1+\alpha}\right)\right. \\
\left.+\frac{\cos [(1-\alpha) \xi]-1}{(1-\alpha)^{2}}-\frac{\cos [(1+\alpha) \xi]-1}{(1+\alpha)^{2}}\right]-\frac{1}{2 \alpha(1+3 \alpha)} \\
\cdot\left\{\sin ^{3}(\alpha \xi) \sin \xi-\frac{1}{1+\alpha}\left[\sin ^{2}(\alpha \xi) \cos [(1-\alpha) \xi]\right.\right. \\
\left.\left.+\alpha\left(\frac{\cos [(1-\alpha) \xi]-1}{1-\alpha}-\frac{\cos [(1-3 \alpha) \xi]-1}{1-3 \alpha}\right)\right]\right\}
\end{gathered}
$$$$
\mathcal{S}_{y}(\xi)=\frac{1}{2}\left[\frac{\cos [(1-\alpha) \xi]-1}{1-\alpha}-\frac{\cos [(1+\alpha) \xi]-1}{1+\alpha}\right],
$$

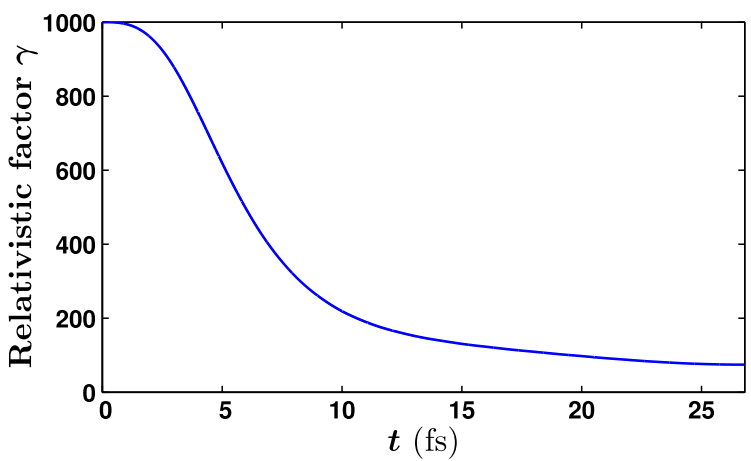

FIG. 12. Relativistic factor $\gamma$ plotted vs time $t$ for a sinusoidalenvelope circularly polarized laser pulse with angular frequency $\omega=2 \times 10^{15} \mathrm{~s}^{-1}$, maximum strength $a_{0}=100$, and a pulsewidth equal to 26.8 fs. The electron has an initial relativistic factor $\gamma_{0}=1000$.

$$
\begin{aligned}
\mathcal{C}_{y}(\xi)= & \frac{1}{4}\left[\xi\left(\frac{\cos [(1-\alpha) \xi]}{1-\alpha}-\frac{\cos [(1+\alpha) \xi]}{1+\alpha}\right)\right. \\
& \left.-\frac{\sin [(1-\alpha) \xi]}{(1-\alpha)^{2}}+\frac{\sin [(1+\alpha) \xi]}{(1+\alpha)^{2}}\right]-\frac{1}{2 \alpha(1+3 \alpha)} \\
& \cdot\left\{\sin ^{3}(\alpha \xi) \cos \xi+\frac{1}{1+\alpha}\left[\sin ^{2}(\alpha \xi) \sin [(1-\alpha) \xi]\right.\right. \\
& \left.\left.+\alpha\left(\frac{\sin [(1-\alpha) \xi]}{1-\alpha}-\frac{\sin [(1-3 \alpha) \xi]}{1-3 \alpha}\right)\right]\right\} . \quad(78 \mathrm{e})
\end{aligned}
$$

The integral for time in Eq. (74) can be evaluated in closed form (not given here) or by computing the simple summation approximating the integral.

With these expressions substituted into Eqs. (64), (67a), and (69a) to obtain $\mathcal{G}_{c}(\xi), \mathcal{H}_{x}(\xi)$, and $\mathcal{H}_{y}(\xi)$, the relativistic factor $\gamma$ and velocities $u_{z} / c$ and $u_{x} / c$ in Eqs. (71)-(73) are plotted in Figs. 12, 13, and 14 for the sinusoidal envelope $\mathcal{E}=\sin (\alpha \xi)$ with $\alpha=0.03169$. This value of $\alpha$ is chosen to give an envelope pulse width of $26.8 \mathrm{fs}$, the length of the uniform-plane-wave pulse chosen in Ref. [17] and the foregoing figures of the present paper. A comparison of these plots in Figs. 12, 13, and 14 with the corresponding plots in Figs. 4, 5, and 6 for the sinusoidal-envelope linearly

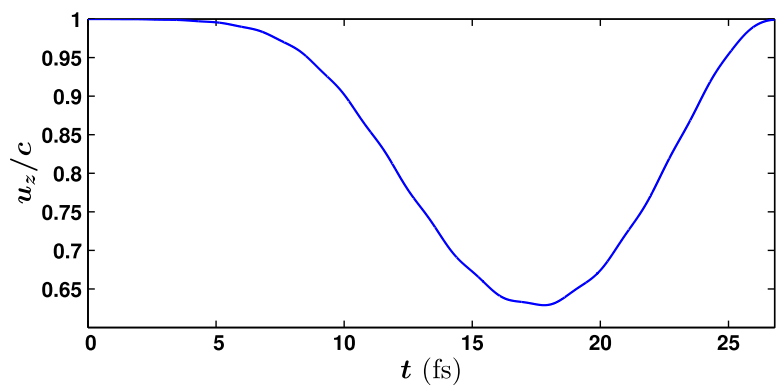

FIG. 13. Longitudinal velocity $u_{z} / c$ plotted vs time $t$ for a sinusoidal-envelope circularly polarized laser pulse with parameters given in Fig. 12. 


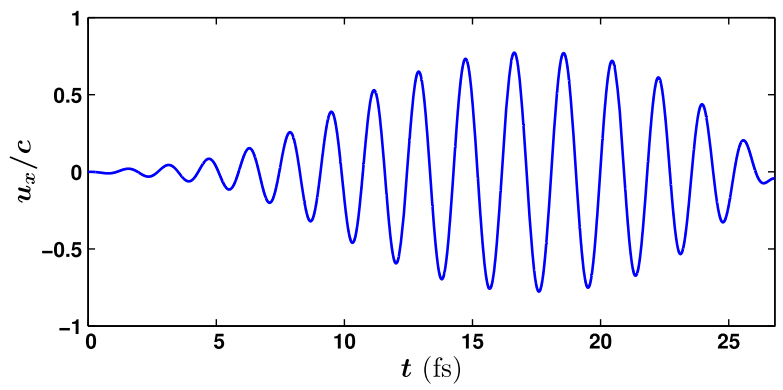

FIG. 14. Transverse velocity $u_{x} / c$ plotted vs time $t$ for a sinusoidal-envelope circularly polarized laser pulse with parameters given in Fig. 12.

polarized plane wave shows that the biggest difference is the smooth variation of $u_{z} / c$ vs time for the circularly polarized sinusoidal-envelope in Fig. 13. Over the $26.8 \mathrm{fs}$ time interval, $\gamma$ remains much greater than unity $(u / c \approx 1)$ for both uniform and sinusoidal circularly polarized plane waves.

The power supplied by the externally applied force to the electron, the power radiated, the kinetic power, and the Schott power, all normalized to $m c^{2}$ and given in units of $s^{-1}$, for the sinusoidal-envelope circular polarization are plotted in Figs. 15 and 16. Again the difference between $P_{\text {rad }}(t)$ and $P_{\text {rad }}^{\mathrm{LAD}}(t)$ (not shown) is smaller than the linewidth of the $P_{\text {rad }}(t)$ curve in Fig. 15, thereby again indicating the high accuracy of the LL solution for the given frequency and strength of the laser beam, and for the initial velocity (relativistic factor) of the electron; see Eq. (52). In addition to all the powers gradually increasing from a value of zero at $t=0$ for the sinusoidal-envelope circular polarization, the main other difference between the powers plotted in Figs. 10 and 11 for the uniform circular polarization and the powers plotted in Figs. 15 and 16 for the sinusoidal-envelope circular polarization is the much smaller value of the Schott power in Fig. 16 for the sinusoidal envelope. Also, the agreement between $P_{\mathrm{Sch}}(t)$ and $P_{\mathrm{Sch}}^{\mathrm{LAD}}(t)$ is not quite as close as in the previous cases of linear polarization and uniform circular polarization, thereby indicating that indeed $P_{\mathrm{Sch}}(t)$ determined by

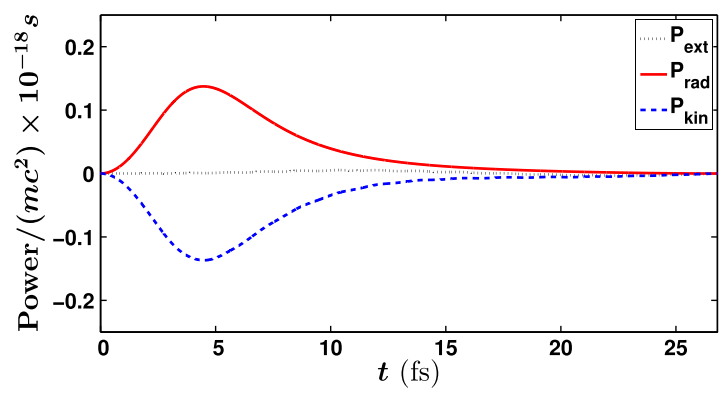

FIG. 15. $\quad P_{\text {ext }}, P_{\text {rad }}$, and $P_{\text {kin }}$ plotted vs time $t$ for a sinusoidalenvelope circularly polarized laser with parameters given in Fig. 12.

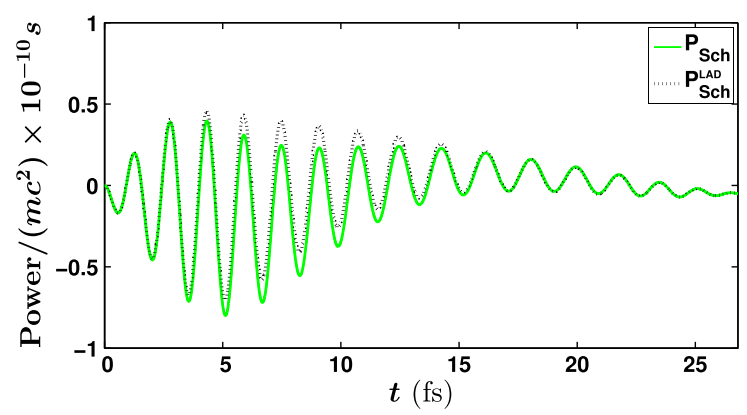

FIG. 16. $P_{\mathrm{Sch}}(t)$ and $P_{\mathrm{Sch}}^{\mathrm{LAD}}(t)$ for the sinusoidal-envelope circularly polarized plane wave with parameters given in Fig. 12.

the LL approximate solution to the LAD equation of motion is not a perfect differential (as the computed integrations over time of both Schott powers confirm).

\section{CONDITIONS FOR THE ACCURACY OF THE LL APPROXIMATE SOLUTION TO THE LAD EQUATION OF MOTION}

The LL solution for the LAD equation of motion is given by the first order successive-substitution approximation derived in Sec. III. Thus, the LL solution is an accurate approximation to the exact solution of the LAD equation of motion if the second and higher-order successive substitution terms are negligible compared to the first term [44]. One way to determine the conditions under which the LL solution is an accurate approximation to the LAD equation of motion is to see if the radiated power in the LAD equation is nearly equal to the radiated power in the LL equation when the LL solution is inserted into the LAD expression for the radiated power in Eq. (51). A convenient way to do this for the problem of the moving electron in a counterpropagating laser beam is to first show that $P_{\text {rad }}^{\mathrm{LAD}}$ in Eq. (51) can be reexpressed as

$$
P_{\mathrm{rad}}^{\mathrm{LAD}}=\tau_{e} \gamma^{2}\left[\left|\frac{d(\gamma \mathbf{u} / c)}{d t}\right|^{2}-\left(\frac{d \gamma}{d t}\right)^{2}\right] .
$$

Then substituting $d(\gamma \mathbf{u} / c) / d t$ from Eq. (27) and $d \gamma / d t$ from Eqs. (29) into (79) for the uniform linearly polarized plane wave, and assuming that

$$
\omega \tau_{e} \gamma\left(1+u_{z} / c\right) \ll 1 \text { say } \lesssim \frac{1}{10},
$$

one finds

$$
\begin{aligned}
P_{\mathrm{rad}}^{\mathrm{LAD}}= & \omega^{2} \tau_{e} a_{0}^{2}\left(1+u_{z} / c\right)^{2} \gamma^{2} \cos ^{2} \xi \\
& \cdot\left[1+\omega^{2} \tau_{e}^{2} a_{0}^{2}\left(1+u_{z} / c\right)^{2} \gamma^{2} \cos ^{2} \xi\right] .
\end{aligned}
$$

For $\omega=2 \times 10^{15} \mathrm{~s}^{-1}$, the inequality in Eq. (80) becomes $\gamma\left(1+u_{z} / c\right) \ll 8 \times 10^{7}$, which is satisfied by electron energies attainable in present-day accelerators. 
The corresponding radiated power given by the LL approximation can be found directly from Eq. (29) to get Eq. (50b), which for the uniform $(\mathcal{E}=1)$ linearly polarized plane wave evaluates to

$$
P_{\mathrm{rad}}=\omega^{2} \tau_{e} a_{0}^{2}\left(1+u_{z} / c\right)^{2} \gamma^{2} \cos ^{2} \xi .
$$

Comparing Eqs. (81) and (82) reveals that the LL approximation to the LAD equation of motion is accurate if and only if

$$
\omega^{2} \tau_{e}^{2} a_{0}^{2}\left(1+u_{z} / c\right)^{2} \gamma^{2} \ll 1 \text { say } \lesssim \frac{1}{10}
$$

or

$$
a_{0} \lesssim \frac{1}{3 \omega \tau_{e} \gamma\left(1+u_{z} / c\right)}
$$

This inequality is the most restrictive when $u_{z} / c \approx 1$, for which it simplifies to

$$
a_{0} \lesssim \frac{1}{6 \omega \tau_{e} \gamma} \approx \frac{1.3 \times 10^{7}}{\gamma} \text { for } \omega=2 \times 10^{15} \mathrm{~s}^{-1} .
$$

However, it should be noted that as $u_{z} / c$ gets close to the value of -1 (which occurs more rapidly the larger the value of $a_{0}$ ), the inequality in Eq. (84) becomes much less restrictive so that the right-hand sides of Eqs. (84)-(85) are multiplied by the large values of $1 /\left(1+u_{z} / c\right)$.

We have obtained the conditions in Eqs. (84) and (85) for the accuracy of the LL approximation by comparing the LL and LAD power equations of motion for the uniform linearly polarized plane wave. Although the proof will not be given here, it can be shown that these same conditions result by comparing the LL and LAD force equations of motion for the uniform linearly polarized plane wave. Also these same conditions derive from the LL and LAD power or force equation of motion for the uniform circularly polarized plane wave. In the rest frame of the electron, the accuracy conditions in Eqs. (80) and (83) reduce to those in Eqs. (19) and (25) derived in Sec. III A for the rest-frame LAD and LL plane-wave equations. The border of the inequality in Eq. (85) is plotted with the dashed blue line in Fig. 17 for $\omega=2 \times 10^{15} \mathrm{~s}^{-1}$.

The condition in Eq. (84) for the LL approximation to be an accurate solution to the LAD equation of motion has the same inverse relationship between $a_{0}$ and $\gamma$ as the condition in Eq. (101b) for the quantum recoil effects of the electron to be significant, except that the constant in Eq. (84) is three orders of magnitude larger than the constant in Eq. (101b). This means that, for the problem of the electron in a counterpropagating laser beam, the LL solution is a highly accurate approximation to the LAD equation of motion unless the value of the product $a_{0} \gamma$ is so large that quantum effects can dominate and neither the LL or LAD equation of motion are adequate, at least without modification for quantum effects (see Fig. 17).

\section{THE LF EQUATION OF MOTION}

The LF equation of motion refers to the equation of motion without any of the radiation-reaction terms. It is obtained from either the LAD equation of motion in Eq. (10) or the LL equation of motion in Eq. (14) by setting $\tau_{e}=0$. One can see from Eqs. (31) and (38) with a uniform plane wave $(\mathcal{E}=1)$ that, because $|\mathcal{C}(\xi)|$ is no larger in value than about $\mathcal{F}(\xi)$, the LL solution becomes approximately equal to the LF solution if the following two conditions are satisfied in the LL solution

$$
\begin{gathered}
\omega \tau_{e} \gamma_{z 0} \ll 1 \text { and } \\
\omega \tau_{e} \gamma_{z 0} a_{0}^{2} \mathcal{F}(\xi) \ll 1
\end{gathered}
$$

Since $\mathcal{F}(\xi)$ can be approximated by $\xi / 2=\omega(t+z / c) /$ 2 , the second condition can be rewritten as

$$
\omega^{2} \tau_{e} \gamma_{z 0} a_{0}^{2}(t+z / c) \ll 1 .
$$

If one writes $\omega(t+z / c)=2 \pi(t+z / c) / T=2 \pi N_{c}$, where $T$ is the period of the plane wave and $N_{c}$ is the number of plane-wave cycles encountered by the moving electron, then Eq. (86c) can be recast as $\omega \tau_{e} \gamma_{z 0} a_{0}^{2} N_{c} \ll 1$ [18].

The condition in Eq. (86c) reveals that after the laser beam is applied to the electron for a long enough time, the LL solution is never well approximated by the LF solution; that is, the radiation reaction terms always become important after a sufficiently long time $t$. In fact, because $\mathcal{F} \rightarrow \infty$ as $t \rightarrow \infty$, Eq. (31) shows that for the LL solution $\gamma(1+$ $\left.u_{z} / c\right) \rightarrow 0$ and thus $u_{z} / c \rightarrow-1$ (implying $\gamma \rightarrow \infty$ and $\left.u_{x} / c \rightarrow 0\right)$ as $t \rightarrow \infty$, whereas for the LF solution $\left(\tau_{e}=0\right)$, Eq. (31) shows that $\gamma\left(1+u_{z} / c\right)=\gamma_{z 0}$ for all time. This implies, for example, that the linear Thomson solution, which has a $u_{x} / c$ that oscillates with a constant magnitude, always becomes invalid after the electron has been subject to the plane wave for a long enough time. These conclusions hold as well for the circularly polarized plane wave with $\mathcal{F}_{c}(\xi)$ replacing $\mathcal{F}(\xi)$ in Eq. (86b). The inequality in Eq. (86a) can be rewritten for $\omega=$ $2 \times 10^{15} \mathrm{~s}^{-1}$ as $\gamma_{z 0} \ll 8 \times 10^{7}$, which is satisfied by electron energies attainable in present-day accelerators.

\section{QUANTUM EFFECTS}

Except for the ad hoc renormalization of the mass of the charged particle as its radius is allowed to approach zero, the LAD equation of motion (10) with its approximate LL solution in Eq. (14) is derived entirely from the classical Maxwell equations and the classical relativistically generalized Newtonian equations of momentum and energy of a mass subject to a force. However, depending on the laser 
intensity and frequency, and the energy of the electron, three different quantum effects can significantly alter the motion and radiation from the charged particle (electron): quantum vacuum electron-positron pair production, Compton scattering of the incident photons, and electron quantum recoil from photon emission ("inverse Compton scattering") $[1,45,46]$.

\section{A. Quantum vacuum electron-positron pair production}

One of the quantum effects involves electron-positron pair production from the quantum vacuum (mentioned in Sec. II), that can occur when the magnitude of the electric field (in the absence of a magnetic field) approaches the Schwinger critical electric field given by

$$
E_{s}=\frac{m^{2} c^{3}}{e \hbar}=1.32 \times 10^{18} \mathrm{~V} / \mathrm{m},
$$

where $\hbar=1.05 \times 10^{-34} \mathrm{~J} \cdot \mathrm{s}$ is the reduced Planck's constant (Planck's constant divided by $2 \pi$ ). As pointed out in footnote 3 , when both magnetic and electric fields are present, electron-positron pair production depends on electromagnetic field invariants that are zero in plane waves (like those in the laser beam). Thus, direct quantum-vacuum pair-production is not possible for a single laser beam regardless of its intensity [1].

\section{B. Compton scattering}

The other two quantum effects depend upon the size of the laser-strength parameter $a_{0}$. To explain these effects, consider the average photon density $n_{p h}$ in the plane wave of the laser beam as seen in the rest frame of the electron. Specifically, equating the average energy density of the photons to the average energy density in the classical planewave fields, we have

$$
n_{p h} \hbar \omega_{0}=\frac{1}{4}\left(\epsilon_{0} E_{0}^{2}+B_{0}^{2} / \mu_{0}\right)=\frac{1}{2} \epsilon_{0} E_{0}^{2},
$$

so that

$$
n_{p h}=\frac{\epsilon_{0} E_{0}^{2}}{2 \hbar \omega_{0}} .
$$

The average distance between the photons is $d_{p h}=1 /\left(n_{p h}\right)^{\frac{1}{3}}$. If this average photon separation distance is too large, then classical electromagnetic field analysis of the electron in a plane wave (linear and nonlinear Thomson scattering) will not suffice and a quantum approach taking into account the Compton scattering between individual photons and the electron is required. The critical separation distance, beyond which classical field theory may fail and quantum theory may be required, can be determined from the classical estimate of the effective size of a photon as the smallest possible quasimonochromatic electromagnetic wave packet that can propagate in free space. The dimensions of this minimum-size free-space classical quasimonochromatic wave packet with frequency $\omega_{0}$ in the rest frame of the electron are approximately $\lambda_{0}$ in the propagation direction and $\lambda_{0} / 2$ in the transverse direction [47]. Thus, these classical photons will create a uniform classical electromagnetic field in free space as seen by the electron if $d_{p h} \ll \lambda_{0}$, say $d_{p h} \lesssim \lambda_{0} / 4$, where $\lambda_{0}$ is the wavelength of the laser in the rest frame of the electron. ${ }^{5}$ Consequently, it seems reasonable to assume that the inequality $d_{p h} \gtrsim \lambda_{0} / 4$ gives the criterion for the rest-frame point charge (electron) to leave the homogeneous continuum regime of the classical Maxwellian electromagnetic fields and to encounter significant quantum Compton scattering effects produced by the individual incident photons. This inequality can be expressed in terms of the laser strength $a_{0}$ and the ratio of the energy of the photon to the rest energy of the electron, $\rho=\hbar \omega_{0} /\left(m c^{2}\right)$ with $\omega_{0}=2 \pi c / \lambda_{0}$, by rewriting $d_{p h}$ in terms of $n_{p h}$ in (89) so that the inequality $d_{p h} \gtrsim \lambda_{0} / 4$ becomes

$$
n_{p h} \lesssim \frac{64}{\lambda_{0}^{3}},
$$

which can be reexpressed as

$$
a_{0} \lesssim(8 \alpha)^{\frac{1}{2}} \rho \approx \frac{\rho}{4},
$$

where $a_{0}$ is given in Eq. (28b) and $\alpha$ is the dimensionless fine-structure constant

$$
\alpha=\frac{e^{2}}{4 \pi \epsilon_{0} \hbar c} \approx \frac{1}{137}
$$

For $a_{0} \gtrsim(8 \alpha)^{\frac{1}{2}} \rho$, the individual photons coalesce to exhibit a classical Maxwellian field. Since the longitudinal Doppler shift of the plane-wave frequency with respect to the counterpropagating relativistic electron gives $\omega_{0} \approx \gamma\left(1+u_{z} / c\right) \omega$, the normalized rest-frame photon energy $\rho$ can be rewritten in terms of the laboratory-frame laser-beam plane-wave frequency $\omega$ as $[11,45]$

$$
\rho=\frac{\gamma\left(1+u_{z} / c\right) \hbar \omega}{m c^{2}}=2.6 \times 10^{-6} \gamma\left(1+u_{z} / c\right),
$$

\footnotetext{
${ }^{5}$ The inequality $d_{p h} \ll \lambda_{0}$ is identical to the condition (in SI units) obtained from quantum electrodynamics by Berestetskii, Lifshitz, and Pitaevskii for the "averaged [quantum electrodynamic] field to be quasi-classical" [[48] Eq. (5.2)]. Note that this condition for the plane-wave fields to behave classically depends only on the average energy density in the classical fields, the frequency in the rest frame of the electron, and Planck's constant. The charged particle (electron) comes into play only through its relativistic factor needed to obtain the rest-frame frequency in terms of the laboratory-frame frequency.
} 
for $\omega=2 \times 10^{15} \mathrm{~s}^{-1}$, so that $\rho$ is never larger than order 1 for the largest relativistic factors of about $10^{5}$ obtainable by the largest present-day accelerators. The criterion in Eq. (91) for requiring Compton scattering can be written as

$a_{0} \lesssim \frac{1}{4}\left(\frac{\hbar \omega}{m c^{2}}\right) \gamma\left(1+u_{z} / c\right) \approx 6.4 \times 10^{-7} \gamma\left(1+u_{z} / c\right)$,

for $\omega=2 \times 10^{15} \mathrm{~s}^{-1}$. Even if $\gamma$ were as large as $10^{5}$, this implies that quantum Compton effects would not be significant until $a_{0}$ were less than about 0.1 . The inequality in Eq. (94) can be rewritten as

$$
\rho \gtrsim 4 a_{0},
$$

which indicates that for a given value of $a_{0}$ the Compton quantum effects increase with the parameter $\rho$, that is, increase with the rest-frame frequency [45]. As an aside, it is noted that the Compton scattering formulas show that for $\rho \ll 1$, the fractional change in the rest-frame electron energy produced by a collision with a photon is proportional to $\rho^{2}$ [[49] Chap. 23, Sec. 1].

Classical linear Thomson scattering [[50] Sec. 14.8] requires $a_{0} \ll 1$ to keep the velocity and acceleration of the electron low enough that the magnetic-field and radiationreaction forces are negligible; see qualifications in Sec. VI. (Nonlinear Thomson scattering does not require the condition $a_{0} \ll 1$.) However, the criterion in Eq. (91) shows that, depending on the value of $\rho$ (that is, rest-frame frequency), there is a small-value threshold for $a_{0}$, below which the scattering of the incident field by the electron cannot be treated classically. In other words, classical linear Thomson scattering applies to plane-wave incident fields with $a_{0}$ in the range

$$
\frac{\rho}{4} \lesssim a_{0} \ll 1
$$

which also implies that $\rho \ll 1$ for linear Thomson scattering. This is confirmed by the fact that for a fixed nonzero value of $\rho$, the Klein-Nishina cross section for Compton scattering is not equal to the linear Thomson scattering cross section regardless of how small the value of $a_{0}$ (both cross sections are independent of $a_{0}$ ), and that the differences are larger for larger values of $\rho$. Only for $\rho \ll$ 1 does the value of the Klein-Nishina cross section approximately equal the value of the linear Thomson cross section [[51,49] Chap. 23, Sec. 2].

\section{Electron quantum recoil from photon emission}

If the photon density is large enough that the fields behave classically, specifically from (91)

$$
a_{0} \gtrsim(8 \alpha)^{\frac{1}{2}} \rho \approx \frac{\rho}{4}
$$

then the plane-wave fields incident on the electron can be considered to be classical Maxwellian electromagnetic fields $\mathbf{E}$ and $\mathbf{B}$ such as those given in Eqs. (26a) and (26b). Under this condition in Eq. (97), the maximum magnitude of the force on the electron in its rest frame is simply $e E_{\mathrm{rf}}$, where $E_{\mathrm{rf}}$ is the amplitude of the plane-wave electric field in the rest frame of the electron.

As the classical rest-frame field exerts a force of magnitude $e E_{\mathrm{rf}}$ on the electron, the accelerating electron emits radiation and experiences a radiation-reaction force. If the energy of the emitted photons is a sizable fraction of the rest energy of the electron, then the quantum recoil effects of the electron produced by the photons may be substantial and an entirely classical solution may not be adequate [52]. The quantum energy of each photon or bundle of photons emitted by the accelerating electron in its rest frame would be expected to occur over the characteristic quantum interaction distance of the rest-frame electron, namely a distance equal to about the Compton wavelength $\lambda_{c}$ of the electron. In that quantum distance, the electric field imparts an energy to the electron equal to

$$
e E_{\mathrm{rf}} \lambda_{c}=\frac{e E_{\mathrm{rf}} \hbar}{m c}=\hbar \omega_{0} a_{0} \quad \text { and } \quad a_{0}=\frac{e E_{\mathrm{rf}}}{m c \omega_{0}} .
$$

Thus, the quantum recoil effects of the electron will be insignificant and a classical nonlinear Thomson solution will suffice if this quantum bundle of photon energy is less than a small fraction of the rest energy of the electron, that is, if $\hbar \omega_{0} a_{0} /\left(m c^{2}\right) \ll 1$. This dimensionless parameter is commonly denoted by $\chi$, so that

$$
\chi=\frac{\hbar \omega_{0} a_{0}}{m c^{2}}=\rho a_{0}=\frac{E_{\mathrm{rf}}}{E_{s}} \ll 1
$$

where $E_{s}$ is the Schwinger critical electric field given in Eq. (87). Conversely, if the amplitude of the plane-wave electric field $E_{\mathrm{rf}}$ applied to the electron in its rest frame is a significant fraction of the Schwinger critical electric field, then quantum recoil effects will generally be significant. The value of this dimensionless ratio $\chi$ is thus a measure of the importance of the quantum recoil effects of the emitted photons on the electron (and the inadequacy of a purely classical solution). It can be rigorously derived from quantum electrodynamics as the parameter that determines the importance of quantum nonlinear effects [46].

Again, from the Doppler shift for a counterpropagating relativistic electron, $\omega_{0} \approx \gamma\left(1+u_{z} / c\right) \omega$ and since $a_{0}$ is a Lorentz invariant, one can write $\chi$ from Eq. (99) in laboratory-frame parameters as

$$
\chi=\frac{\gamma\left(1+u_{z} / c\right) \hbar \omega a_{0}}{m c^{2}}=\rho a_{0} .
$$

If one chooses (somewhat arbitrarily) $\chi \gtrsim 1 / 10$ as the inequality for determining significant electron quantum 
recoil effects of the emitted photons, Eq. (100) provides the criterion for the need to include these quantum effects as

$$
a_{0} \gtrsim \frac{m c^{2}}{10 \gamma\left(1+u_{z} / c\right) \hbar \omega}=\frac{1}{10 \rho} .
$$

Since the reduced Planck's constant can be expressed in terms of the fine-structure constant $\alpha$ in Eq. (92) and the time constant $\tau_{e}$ in Eq. (8) as $\hbar=3 m c^{2} \tau_{e} /(2 \alpha)$, the criterion in Eq. (101a) for requiring quantum emission recoil effects can be reexpressed as

$$
a_{0} \gtrsim \frac{\alpha}{15 \gamma\left(1+u_{z} / c\right) \omega \tau_{e}} \approx \frac{4 \times 10^{4}}{\gamma\left(1+u_{z} / c\right)},
$$

for $\omega=2 \times 10^{15} \mathrm{~s}^{-1}$. The rest-frame condition (99) for negligible quantum recoil effects can be reexpressed as

$$
\omega_{0} \tau_{e} a_{0} \lesssim \frac{\alpha}{15} \approx 5 \times 10^{-4}
$$

a condition that also implies the inequality in Eq. (25) is satisfied for the LL approximation to be an accurate solution to the LAD equation of motion.

\section{Regions of validity of the different solutions}

The borders of the criteria in Eqs. (101) and (94) for quantum recoil effects of the emitted photons and quantum Compton scattering of the incident photons are plotted by the solid red and dotted black lines, respectively, in Fig. 17 for a laser beam with a frequency $\omega=2 \times 10^{15} \mathrm{~s}^{-1}$ and $u_{z} / c \approx 1$. Above the solid red line and dotted black line, the LAD equation of motion and its LL approximation may not adequately represent an accurate solution for the electron in

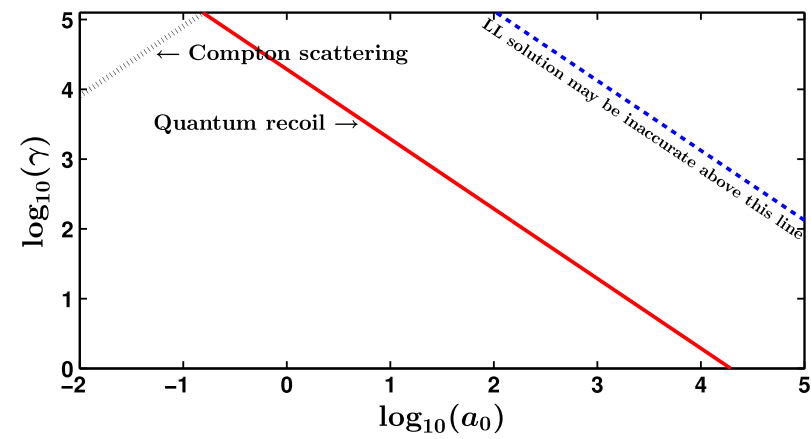

FIG. 17. The $\log _{10}(\gamma)$ vs $\log _{10}\left(a_{0}\right)$ plot of the regions of validity for the relativistic electron in a counterpropagating laser beam with $\omega=2 \times 10^{15} \mathrm{~s}^{-1}$ and $u_{z} / c \approx 1$. Above the solid red line, quantum recoil effects of the emitted photons may be significant. Above the dotted black line, quantum Compton scattering of the individual incident photons may be significant. Above the dashed blue line, the LL solution may not be an accurate approximation to the LAD solution. a counterpropagating laser beam without incorporating quantum effects. Note that the vertical axis in Fig. 17 is $\log _{10}[\gamma(t)]$ not $\log _{10}\left(\gamma_{0}\right)$, where $\gamma_{0}=\gamma(0)$ is the initial value of $\gamma$. Thus, quantum effects may be substantial at $\gamma_{0}$ when the electron first encounters the laser pulse but become negligible when $\gamma$ falls well below $\gamma_{0}$. Martins et al. [11] find substantial quantum corrections for $\gamma_{0}=$ 1030 and $a_{0} \gtrsim 10$, values that lie reasonably close to the red line in Fig. 17.

The border of the criterion in Eq. (85) for the LL approximation in Eq. (14) to be an accurate solution to the LAD equation of motion in Eq. (10) for a relativistic electron $\left(u_{z} / c \approx 1\right)$ in a counterpropagating laser beam is given by the dashed blue line in Fig. 17. Above this dashed blue line, the LL solution may not agree well with the LAD solution. The numerical examples in the present paper for $a_{0}=100$ and initial $\gamma_{0}=1000$ lie well below the dashed blue line, even though this high value of $a_{0} \gamma$ produces measurable $\gamma$-ray frequencies in the radiation emitted by the decelerating electron $[7,8]$.

The solid red line and the dashed blue line are parallel but separated by a large space. This means that the LL approximate solution to the LAD equation of motion becomes inaccurate only well into the region where quantum recoil effects can dominate the solution. This result confirms the commonly held idea that wherever the LL solution fails, the quantum effects will be so strong that the LAD solution will also fail [53-55]. It also justifies the use of the LL approximate solution to the LAD equation of motion as a starting solution for incorporating quantum effects perturbatively [11], as long as the solution lies below the dashed blue line in Fig. 17. Above the dashed blue line where the LL approximation fails, it is conceivable that the LAD equation of motion, if numerically solvable, could provide the starting solution for incorporating quantum effects.

\section{SUMMARY AND CONCLUSIONS}

The root cause of the noncausality (preacceleration/ deceleration) in the LA equation of motion for an extended charge is determined and eliminated by including transition-force contributions at the points in time where the external force is a nonanalytic function of time, such as when the external force is first applied and when it is terminated. If the radius of the extended charge is allowed to approach zero and the associated unbounded mass is renormalized to a finite value (typically the mass of the electron), all the unwanted higher order terms in this causal LA equation of motion vanish, resulting in a causal LAD equation of motion. However, this causal LAD equation of motion is not entirely satisfactory since it turns out that the renormalization of the mass introduces a possible violation of momentum-energy conservation if (and only if) the change in the externally applied force across a transition interval is enormously large (two orders of magnitude 
larger than the force that would be exerted on the charge by the Schwinger electric field needed to produce quantumvacuum electron-positron pairs). Renormalization of the mass in the classical equation of motion is not an entirely innocuous, seamless procedure.

Consequently, since it is also true that the transition forces required for causality have a negligible effect on the LAD solution except for the very short time equal to about $\tau_{e}=6.27 \times 10^{-24} \mathrm{~s}$ (for an electron) near the transition points, one can ignore the transition forces with impunity and use the traditional noncausal LAD equation of motion that predicts preacceleration/deceleration. Moreover, the LL approximate solution to the LAD equation of motion does not exhibit noncausal preacceleration/deceleration because the LL solution is nothing more than the firstorder term of a successive-substitution power-series solution to the LAD equation of motion.

A transparent, convenient three-vector formulation of the LL approximation is used to obtain its exact closed-form solution for an electron moving in an arbitrarily shaped counterpropagating plane-wave laser pulse. Numerical results are plotted for the velocity of the electron and for its various powers (radiated, kinetic, externally applied, and Schott) as functions of time for linearly and circularly polarized, rectangular-envelope and sinusoidal-envelope plane-wave pulses with a laser-strength parameter $a_{0}=$ 100 and an initial electron relativistic factor $\gamma_{0}=1000$. Explicit expressions are found for the time $t$ in terms of the retarded time parameter $\xi$. The sinusoidal envelopes are shown to strongly reduce the rapid changes in velocity and power that occur near the beginning of the rectangularenvelope pulses. Analytical and numerical agreement is found with previously published results for the velocity.

General conditions are obtained for the LL approximation to be an accurate solution to the LAD equation of motion, and for the LL solution to reduce to the LF solution that contains no explicit radiation reaction. It is proven that the LF solution is never adequate if the external force is applied for a long enough time.

Simple semiclassical derivations are used to obtain the parameters and conditions for deciding the importance of both quantum Compton scattering of the electron by the incident photons and the electron quantum recoil effects produced by the emitted photons ("inverse Compton scattering"). These parameters and conditions are shown to be identical to those obtained from quantum electrodynamics by Landau and Lifshitz and by Ritus. It is proven conclusively that the LL approximation becomes an inaccurate solution to the LAD equation of motion only where the product $a_{0} \gamma$ is large enough that quantum recoil effects on the electron can dominate over the classical solution.

\section{ACKNOWLEDGMENTS}

Informative discussions with Professors M. Vranic, A. Di Piazza, and Y. Hadad are gratefully acknowledged.
This research was supported in part under the U.S. Air Force Office of Scientific Research (AFOSR) Grant No. FA9550-19-1-0097 through Dr. A. Nachman.

[1] S. V. Bulanov, T. Z. Esirkepov, Y. Hayashi, M. Kando, H. Kiriyama, J. K. Koga et al., On the design of experiments for the study of extreme field limits in the interaction of laser with ultrarelativistic electron beam, Nucl. Instrum. Methods Phys. Res., Sect. A 660, 31 (2011).

[2] K. T. Phuoc, S. Corde, C. Thaury, V. Malka, A. Tafzi, J. P. Goddet, R. C. Shah, S. Sebban, and A. Rousse, All-optical Compton gamma-ray source, Nat. Photonics 6, 308 (2012).

[3] S. Chen, N. D. Powers, I. Ghebregziabher, C. M. Maharjan, C. Liu, G. Golovin, S. Banerjee, J. Zhang, N. Cunningham, A. Moorti, S.Clarke, S. Pozzi, and D. P. Umstadter, Mevenergy $\mathrm{X}$ rays from Inverse Compton Scattering with Laser-Wakefield Accelerated Electrons, Phys. Rev. Lett. 110, 155003 (2013).

[4] N. D. Powers, I. Ghebregziabher, G. Golovin, C. Liu, S. Chen, S. Banerjee, J. Zhang, and D. P. Umstadter, Quasimonoenergetic and tunable X-rays from a laser-driven Compton light source, Nat. Photonics 8, 28 (2014).

[5] G. Sarri, D. J. Corvan, W. Schumaker, J. M. Cole, A. Di Piazza, H. Ahmed, C.Harvey, C. H. Keitel, K. Krushelnick, S. P. D. Mangles, Z. Najmudin, D. Symes, A. G. R. Thomas, M. Yeung, Z. Zhao, and M. Zepf, Ultrahigh Brilliance Multi-MeV $\gamma$-ray Beams from Nonlinear Relativistic Thomson Scattering, Phys. Rev. Lett. 113, 224801 (2014).

[6] K. Khrennikov, J. Wenz, A. Buck, J. Xu, M. Heigoldt, L. Veisz, and S. Karsch, Tunable All-Optical Quasimonochromatic Thomson X-ray Source in the Nonlinear Regime, Phys. Rev. Lett. 114, 195003 (2015).

[7] J. M. Cole et al., Experimental Evidence of Radiation Reaction in the Collision of a High-Intensity Laser Pulse with a Laser-Wakefield Accelerated Electron Beam, Phys. Rev. X 8, 011020 (2018).

[8] K. Poder et al., Experimental Signatures of the Quantum Nature of Radiation Reaction in the Field of an Ultraintense Laser, Phys. Rev. X 8, 031004 (2018).

[9] M. Vranic, T. Grismayer, S. Meuren, R. A. Fonseca, and L. O. Silva, Are we ready to transfer optical light to gamma-rays?, Phys. Plasmas 26, 053103 (2019).

[10] R. Lieu and W. I. Axford, Synchrotron radiation: An inverse Compton effect, Astrophys. J. 416, 700 (1993).

[11] J. L. Martins, M. Vranic, T. Grismayer, J. Vieira, R. A. Fonseca, and L. O. Silva, Modelling radiation emission in the transition from the classical to the quantum regime, Plasma Phys. Controlled Fusion 58, 014035 (2016).

[12] M. Vranic, J. L. Martins, R. A. Fonseca, and L. O. Silva, Classical radiation reaction in particle-in-cell simulations, Comput. Phys. Commun. 204, 141 (2016).

[13] M. Vranic, J. L. Martins, J. Vieira, R. A. Fonseca, and L. O. Silva, All-Optical Radiation Reaction at $10^{21} \mathrm{~W} / \mathrm{cm}^{2}$, Phys. Rev. Lett. 113, 134801 (2014).

[14] T. G. Blackburn, Radiation reaction in electron-beam interactions with high-intensity lasers, Rev. Mod. Plasma Phys. 4, 1 (2020). 
[15] A. Di Piazza, Exact solution of the Landau-Lifshitz equation in a plane wave, Lett. Math. Phys. 83, 305 (2008).

[16] A. Di Piazza, K. Z. Hatsagortsyan, and C. H. Keitel, Strong Signatures of Radiation Reaction Below the RadiationDominated Regime, Phys. Rev. Lett. 102, 254802 (2009).

[17] Y. Hadad, L. Labun, J. Rafelski, N. Elkina, C. Klier, and H. Ruhl, Effects of radiation reaction in relativistic laser acceleration, Phys. Rev. D 82, 096012 (2010).

[18] M. Ruijter, V. Yu. Karin, and S. G. Rykovanov, Analytical solutions for nonlinear Thomson scattering including radiation reaction, J. Phys. B 51, 225701 (2018).

[19] A. Di Piazza, K. Z. Hatsagortsyan, and C. H. Keitel, Quantum Radiation Reaction Effects in Multiphoton Compton Scattering, Phys. Rev. Lett. 105, 220403 (2010).

[20] N. Neitz and A. Di Piazza, Stochasticity Effects in Quantum Radiation Reaction, Phys. Rev. Lett. 111, 054802 (2013).

[21] T. G. Blackburn, C. P. Ridgers, J. G. Kirk, and A. R. Bell, Quantum radiation reaction in laser-electron-beam collisions, Phys. Rev. Lett. 112, 015001 (2014).

[22] S. R. Yoffe, Y. Kravets, A. Noble, and D. A. Jaroszynski, Longitudinal and transverse cooling of relativistic electron beams in intense laser pulses, New J. Phys. 17, 053025 (2015).

[23] M. Vranic, T. Grismayer, R. A. Fonseca, and L. O. Silva, Quantum radiation reaction in head-on laser-electron beam interaction, New J. Phys. 18, 073035 (2016).

[24] F. Niel, C. Riconda, F. Amiranoff, R. Duclous, and M. Grech, From quantum to classical modeling of radiation reaction: A focus on stochasticity effects, Phys. Rev. E 97, 043209 (2018).

[25] H. A. Lorentz, La theorie electromagnetique de Maxwell et son application aux corps mouevemants, Arch. Neerl. Sci. Exactes Nat. 25, 363 (1892).

[26] J.Z. Buchwald, From Maxwell to Microphysics (University of Chicago Press, Chicago, 1985).

[27] M. Abraham, Theorie der Elektrizitat, Elektromagnetische Theorie der Strahlung Vol II (Teubner, Leipzig, 1905).

[28] A. D. Yaghjian, Relativistic Dynamics of a Charged Sphere: Updating the Lorentz-Abraham model, 2nd ed. (Springer, New York, 2006).

[29] A. D. Yaghjian, Absence of a consistent classical equation of motion for a mass-renormalized point charge, Phys. Rev. E 78, 046606 (2008).

[30] T. Rothman, The curse of $E=m c^{2}$, Am. Sci. 109, 360 (2021).

[31] J. Schwinger, Electromagnetic mass revisited, Found. Phys. 13, 373 (1983).

[32] G. A. Schott, On the motion of the Lorentz electron, Philos. Mag. 29, 49 (1915).

[33] G. A. Schott, Über den Einfluss von Unstetigkeiten bei der Bewegung von Elektronen, Ann. Phys. (Berlin) 330, 63 (1908).

[34] G. A. Schott, Electromagnetic Radiation (Cambridge University Press, Cambridge, 1912).

[35] A. Valentini, Resolution of Causality Violation in the Classical Radiation Reaction, Phys. Rev. Lett. 61, 1903 (1988).
[36] C. J. Goebel, Comment on "Resolution of Causality Violation in the Classical Radiation Reaction", Phys. Rev. Lett. 62, 3010 (1989).

[37] P. A. M. Dirac, Classical theory of radiating electrons, Proc. R. Soc. A 167, 148 (1938).

[38] S. S. Bulanov, Pair production by a circularly polarized electromagnetic wave in a plasma, Phys. Rev. E 69, 036408 (2004).

[39] S. V. Bulanov, T. Zh. Esirkepov, M. Kando, J. K. Koga, and S.S. Bulanov, Lorentz-Abraham-Dirac versus LandauLifshitz radiation friction force in the ultrarelativistic electron interaction with electromagnetic wave (exact solutions), Phys. Rev. E 84, 056605 (2011).

[40] L. D. Landau and E. M. Lifshitz, The Classical Theory of Fields, 4th ed., (Pergamon, Oxford, 1975).

[41] A. G. R. Thomas, C. P. Ridgers, S. S. Bulanov, B. J. Griffin, and S. P. D. Mangles, Strong Radiation-Damping Effects in a Gamma-Ray Source Generated by the Interaction of a High-Intensity Laser with a WakefieldAccelerated Electron Beam, Phys. Rev. X 2, 041004 (2012).

[42] M. R. Spiegel, Applied Differential Equations, 3rd ed. (Pearson, New York, 1980).

[43] T. Schlegel and V. T. Tikhonchuk, Classical radiation effects on relativistic electrons in ultraintense laser fields with circular polarization, New J. Phys. 14, 073034 (2012).

[44] R. R. Pandit and Y. Sentoku, Higher order terms of radiative damping in extreme intense laser-matter interaction, Phys. Plasmas 19, 073304 (2012).

[45] F. Mackenroth and A. Di Piazza, Nonlinear Compton scattering in ultrashort laser pulses, Phys. Rev. A 83, 032106 (2011).

[46] V. I. Ritus, Quantum effects of the interaction of elementary particles with an intense electromagnetic field, J. Sov. Laser Res. 6, 497 (1985).

[47] A. D. Yaghjian, Stellar speckle and correlation functions derived from classical wave expansions for spherical antennas, IEEE Trans. Antennas Propag. 68, 7423 (2020).

[48] V. B. Berestetskii, E. M. Lifshitz, and L. P. Pitaevskii, Quantum Electrodynamics, 2nd ed. (Pergamon, Oxford, 1982).

[49] R. D. Evans, The Atomic Nucleus (McGraw-Hill, New York, 1955).

[50] J. D. Jackson, Classical Electrodynamics, 3rd ed. (John Wiley, New York, 1999).

[51] O. Klein and Y. Nishina, On the scattering of radiation by free electrons according to Dirac's new relativistic quantum dynamics, in The Oskar Klein Memorial Lectures, edited by G. Ekspong (World Scientific, Singapore, 1994), Vol. 2, pp. 113-129.

[52] A. Ilderton and G. Torgrimsson, Radiation reaction in strong field QED, Phys. Lett. B 725, 481 (2013).

[53] F. Rohrlich, Dynamics of a classical quasi-point charge, Phys. Lett. A 303, 307 (2002).

[54] C. S. Shen, Comment on the "new" equation of motion for classical charged particles, Phys. Rev. D 6, 3039 (1972).

[55] C. S. Shen, Radiation and acceleration of a relativistic charged particle in an electromagnetic field, Phys. Rev. D 17, 434 (1978). 